\title{
Displacement of fluid droplets from solid surfaces in low-Reynolds-number shear flows
}

\author{
By P. DIMITRAKOPOULOS AND J. J. L. HIGDON \\ Department of Chemical Engineering, University of Illinois, Urbana, IL 61801, USA
}

(Received 18 July 1996 and in revised form 18 November 1996)

The yield conditions for the displacement of fluid droplets from solid boundaries are studied through a series of numerical computations. The study includes gravitational and interfacial forces, but is restricted to two-dimensional droplets and low-Reynoldsnumber flow. A comprehensive study is conducted, covering a wide range of viscosity ratio $\lambda$, Bond number $B_{d}$, capillary number $C a$ and contact angles $\theta_{A}$ and $\theta_{R}$. The yield conditions for drop displacement are calculated and the critical shear rates are presented as functions $C a\left(\lambda, B_{d}, \theta_{A}, \Delta \theta\right)$ where $\Delta \theta=\theta_{A}-\theta_{R}$ is the contact angle hysteresis. The numerical solutions are based on the spectral boundary element method, incorporating a novel implementation of Newton's method for the determination of equilibrium free surface profiles. The numerical results are compared with asymptotic theories (Dussan 1987) based on the lubrication approximation. While excellent agreement is found in the joint asymptotic limits $\Delta \theta \ll \theta_{A} \ll 1$, the useful range of the lubrication models proves to be extremely limited. The critical shear rate is found to be sensitive to viscosity ratio with qualitatively different results for viscous and inviscid droplets. Gravitational forces normal to the solid boundary have a significant effect on the displacement process, reducing the critical shear rate for viscous drops and increasing the rate for inviscid droplets. The low-viscosity limit $\lambda \rightarrow 0$ is shown to be a singular limit in the lubrication theory, and the proper scaling for $\mathrm{Ca}$ at small $\lambda$ is identified.

\section{Introduction}

The displacement of liquid droplets from solid substrates is a fundamental problem of fluid mechanics. This problem has application in numerous areas including distillation, spray coating, packed towers and a variety of multiphase flow operations in the chemical process industry. Our interest in the problem focuses on drop displacement in viscous shear flows at low Reynolds number. This regime has relevance in coating operations and in enhanced oil recovery. In the coating industry, the presence of small liquid droplets or gas bubbles on solid surfaces is a major concern in the design of process equipment, because even a small flow disturbance is sufficient to destroy the uniformity required in precision film coating. In the petroleum industry, enhanced oil recovery techniques are strongly dependent on the interaction of oil and water in immiscible two-phase mixtures, and the success of such operations depends on the displacement of small oil droplets attached to solid surfaces.

The fundamental issues associated with viscous drop displacement from rigid boundaries have been addressed in a series of papers by Dussan and coworkers (Dussan \& Chow 1983; Dussan 1985, 1987). These authors developed yield criteria 
for the critical capillary number $\mathrm{Ca}$ as a function of the advancing and receding contact angles, $\theta_{A}$ and $\theta_{R}$. In the first two articles, they considered droplets in a quiescent fluid displaced by the action of gravity. In the final article, Dussan considered the displacement of a droplet through the action of a viscous shear flow. In each of these papers, the analysis was based on asymptotic theory valid for small contact angle hysteresis $\left(\theta_{A}-\theta_{R}\right)$. Additional simplifications included the restriction to small contact angles and lubrication theory in the first and third articles and to small Bond numbers $B_{d}$ in the second article. Johnson (1981) considered a problem analogous to that of Dussan (1987) involving the stability of liquid layers attached to solid spherical particles. He developed a lubrication theory for axisymmetric flows of thin liquid films under the assumption of small contact angles. Additional studies of fluid films enclosing solid particles are discussed in the review article by Johnson \& Sadhal (1985).

At the opposite end of the flow spectrum, Durbin (1988) analysed the displacement of droplets in inviscid flow, employing free streamline theory and an asymptotic analysis for infinitesimal contact angles. King \& Tuck (1993) studied a similar problem for a thin droplet in a nearly inviscid flow. They assumed that the exterior flow induced a constant stress (or drag coefficient) on the drop surface and solved the lubrication equations inside the droplet to determine yield conditions for drops on inclined surfaces. Their model considered a balance between viscous and gravitational forces, ignoring the effects of surface tension. Tuck \& Schwartz (1991) considered droplets on inclined surfaces in the absence of exterior fluids with equilibrium configurations determined by gravitational forces and surface tension.

Feng \& Basaran (1994) conducted a detailed study of the drop displacement problem for steady two-dimensional flows at arbitrary Reynolds number $R e$. These authors utilized finite element solutions of the Navier-Stokes equations to determine the yield conditions for a range of Reynolds number and capillary number and identified the Weber number $W e \equiv \operatorname{Re} C a$ as the most important parameter for drop displacement. In this study, the authors emphasized modest values of the capillary number for which the stress at the critical point was dominated by inertial forces, hence the dependence on Weber number. Feng \& Basaran restricted their computations to bubbles with zero viscosity and no gravitational forces. At low Reynolds number, this work complements that of Dussan and Chow, but is not directly comparable owing to different geometries and assumptions at the contact line. Dussan and Chow analysed three-dimensional droplets attached to a smooth plane with contact angles limited by $\theta_{A}$ and $\theta_{R}$, while Feng \& Basaran considered two-dimensional droplets attached to a slot in a plane wall with the contact line pinned at the sharp boundary edge. Dussan and Chow presented an asymptotic theory valid at small contact angles, while Feng \& Basaran analysed bubbles with large contact angles with the bulk of their results devoted to contact angles of $90^{\circ}$. In a recent paper, Li \& Pozrikidis (1996) studied the three-dimensional analogue of the problem of Feng \& Basaran in the limit of low-Re flow. These authors assumed a contact line of prescribed shape, computed the shapes of droplets as a function of $\mathrm{Ca}$ and reported on the distribution of contact angles around the contact line. Owing to the difficulty of the three-dimensional flow problem, this study was limited to fluids and droplets of equal viscosity and computations were performed for a limited range of parameter values. More significantly, the prescription of contact lines of elliptical planform is an approximation which does not strictly conform to observations (Furmidge 1962) or to the asymptotic theory of Dussan (1987). 
In the present article, we address the two-dimensional analogue of Dussan's problem, but make no restrictions on any parameter values or flow conditions. Our goal is to provide a comprehensive solution of the problem and hence to assess the limits of the asymptotic theories. In particular, we seek to determine the range of validity for lubrication approximations and the effect of fluid viscosity ratio. We include gravitational effects to investigate droplets which feature large aspect ratios and relatively flat interfaces with arbitrary contact angles. In particular, we consider the displacement of a two-dimensional droplet from a plane substrate due to the action of a viscous shear flow in the presence of gravitational forces. We consider arbitrary values for the contact angles, the contact angle hysteresis $\left(\theta_{A}-\theta_{R}\right)$ and the viscosity ratio, and include a range of Bond numbers for gravity acting normal to the plane substrate. We determine the equilibrium shapes of the fluid interface and find the yield condition for drop displacement. In this paper, we define an equilibrium shape to be a stationary interface profile for which all kinematic and dynamic boundary conditions are satisfied under conditions of steady flow. This should not be confused with the concept of equilibrium surfaces under quiescent conditions. The yield condition is expressed as a critical $C a$ as a function of contact angle $\theta_{A}$, hysteresis $\left(\theta_{A}-\theta_{R}\right)$, viscosity ratio $\lambda$ and Bond number $B_{d}$.

To address these problems, we employ the spectral boundary element method described by Occhialini, Muldowney \& Higdon (1992) and further discussed by Muldowney \& Higdon (1995). This algorithm is combined with an iterative scheme to determine the equilibrium shapes of the fluid interface. In prior boundary element studies, most authors have determined equilibrium shapes by time-dependent computations (e.g. Li \& Pozrikidis 1996) or simple iterative strategies with slow convergence rates over small domains (see Pozrikidis 1992). Here we employ a novel approach which implements a Newton iteration scheme within the boundary integral framework. We obtain the rapid convergence characteristic of Newton's method and find a large radius of convergence. The performance of this algorithm compares well with the Newton iteration method of Higdon \& Schnepper (1994), but the implementation is simpler than that of the earlier procedure.

In the analysis of free surface flows, Newton's method has long been established as an effective tool in the finite element literature. The implementation of these algorithms has been discussed in numerous publications by Scriven and coworkers with application to coating flows. Early efforts employing algebraic grid generation are discussed by Saito \& Scriven (1981) and by Kistler \& Scriven (1984a,b), while more recent implementations based on elliptic mesh generation are described by Christodoulou \& Scriven (1992). Additional references for finite element/Newton's method applications in free surface flow are cited in these publications, as well as in the aforementioned work of Feng \& Basaran (1994). In the implementation of a Newton method for boundary integral computations, we seek to extend to this class of algorithms, an effective technique which has long proved its value in the field of finite element computations.

\section{Mathematical formulation}

We consider a two-dimensional droplet attached to a plane solid boundary as illustrated in figure 1 . The droplet size is specified by its volume $V_{0}$ or equivalently by the radius $a$ of a circular droplet of volume $\pi a^{2}=V_{0}$. The droplet (fluid 1) has density $\rho_{1}$ and viscosity $\lambda \mu$, while the surrounding fluid has density $\rho_{2}$ and viscosity $\mu$. The gravitational acceleration is $g$ and points in the negative $y$-direction. The 


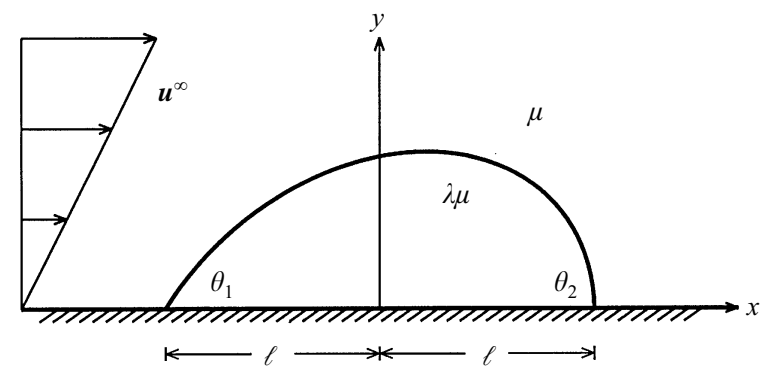

FIGURE 1. Fluid droplet attached to a solid boundary in a viscous shear flow.

surface tension $\gamma$ is assumed constant. The undisturbed flow exterior to the droplet is that of a simple shear flow $\boldsymbol{u}^{\infty}=(G y, 0)$ where $G$ is the shear rate. The contact line intersects the solid wall at positions $\boldsymbol{x}=( \pm \ell, 0)$ with the upstream angle designated $\theta_{1}$ and the downstream angle $\theta_{2}$. Without loss of generality, we have chosen the origin centred between the two contact points. Note that the distance $\ell$ is not fixed but is determined as a part of the problem solution as discussed below.

The capillary number $C a$ and Bond number $B_{d}$ are defined by

$$
C a=\frac{\mu G a}{\gamma}, \quad B_{d}=\frac{\left(\rho_{1}-\rho_{2}\right) g a^{2}}{\gamma \theta_{A}} .
$$

The definition of the Bond number follows from characteristic values for the gravitational forces based on drop height and interfacial forces based on drop curvature. This definition is consistent with that of Dussan and coworkers.

The governing equations in fluid 2 are the Stokes equations together with continuity

$$
\begin{gathered}
\nabla \cdot \boldsymbol{\sigma}=-\nabla p+\mu \nabla^{2} \boldsymbol{u}=0, \\
\nabla \cdot \boldsymbol{u}=0,
\end{gathered}
$$

while in the droplet, the same equations apply with the viscosity replaced by $\lambda \mu$.

The boundary conditions on the solid wall and at infinity give

$$
\begin{array}{ccc}
\boldsymbol{u}=0 & \text { on } & y=0, \\
\boldsymbol{u} \rightarrow \boldsymbol{u}^{\infty} & \text { as } & r \rightarrow \infty .
\end{array}
$$

At the interface, the boundary conditions on the velocity $\boldsymbol{u}$ and surface stress $\boldsymbol{f}$ are

$$
\begin{gathered}
\boldsymbol{u}_{1}=\boldsymbol{u}_{2} \\
\Delta \boldsymbol{f}=\boldsymbol{f}_{2}-\boldsymbol{f}_{1}=\gamma(\nabla \cdot \boldsymbol{n}) \boldsymbol{n}+\left(\rho_{2}-\rho_{1}\right)(\boldsymbol{g} \cdot \boldsymbol{x}) \boldsymbol{n} .
\end{gathered}
$$

Here the subscripts designate quantities evaluated in fluids 1 and 2 respectively. The surface stress is defined as $\boldsymbol{f}=\boldsymbol{\sigma} \cdot \boldsymbol{n}$ and $\boldsymbol{n}$ is the unit normal which we choose to point into fluid 2. The pressure as defined in $\sigma$ is the dynamic pressure, hence the gravity force is absent from (1) and appears in the interfacial stress boundary condition (6).

With an interface of prescribed shape, the equations and boundary conditions (1)(6) constitute a well-posed boundary value problem from which one may determine the velocity and stress throughout both fluid regions. For a droplet profile of arbitrary shape, the solution for the flow field will not in general correspond to a stationary equilibrium shape. For equilibrium shapes, the velocity field must satisfy an additional 
constraint - the kinematic condition at the interface

$$
\boldsymbol{u}_{1} \cdot \boldsymbol{n}=\boldsymbol{u}_{2} \cdot \boldsymbol{n}=0 .
$$

Although the governing equations and boundary conditions are linear in $\boldsymbol{u}$ and $\boldsymbol{f}$, the problem of determining equilibrium droplet shapes constitutes a nonlinear problem for the unknown profile $\Gamma$ : that is the velocity $\boldsymbol{u}$ and stress $\boldsymbol{f}$, as well as the curvature $\nabla \cdot \boldsymbol{n}$ are nonlinear functions of the geometrical variables describing the interface shape. For a given flow field $\boldsymbol{u}^{\infty}$ and droplet volume $V_{0} \equiv \pi a^{2}$, there is no guarantee that an equilibrium shape exists, nor is there any certainty that any solutions are unique. For freely suspended droplets, the system of equations (1)-(7) is sufficient to determine the family of equilibrium profiles. For droplets in contact with a solid boundary, additional conditions are required to prescribe the interface shape in the vicinity of the contact line.

The boundary conditions at the contact line relate the contact angle $\theta$ to the position and velocity of the contact line. A wealth of literature has been devoted to this subject with a review of early work given by Dussan (1979), and a comprehensive review of more recent work given by Kistler (1993). We note that the prediction of dynamic contact angles for moving contact lines poses a far greater challenge than that for the static contact angles required in the present study. We refer the reader to Chen, Rame \& Garoff (1995) for further discussion of recent work in this area.

For a stationary contact line, the boundary condition is determined by the nature of the solid surface. For a rough surface, one may require that the positions of the contact lines remain at fixed positions while allowing the contact angle to vary over a wide range. This is the condition assumed by Feng \& Basaran (1994). From an experimental viewpoint, this condition may be realized by a droplet attached to an orifice or a slit in the plane (Feng \& Basaran 1994; Oliver, Huh \& Mason 1977). At the opposite extreme, one may consider a perfectly smooth, homogeneous surface on which the contact angle takes a single value $\theta$. An elementary force balance (see the Appendix, also Dussan \& Chow 1983) shows that a droplet on a such a surface cannot resist a net force arising from fluid motion; hence stationary droplets could exist only in quiescent fluids. For real surfaces, it has been found that the static contact angle exhibits a hysteresis effect where the contact line remains stationary for any angle in the range $\theta_{R}<\theta<\theta_{A}$. The limits $\theta_{A}$ and $\theta_{R}$ are called the advancing and receding angles respectively.

The source of contact angle hysteresis has been the subject of much study in the literature. Experimental observations (Good \& Koo 1979; Gaydos \& Neumann 1987; Yekta-Fard \& Ponter 1988) show that the hysteresis is a function not only of the fluid and solid properties, but also of the size of the droplets. Recent theories on the origin of these phenomena point to microscopic variation in surface properties, either in the form of surface roughness or chemical inhomogeneity (Marmur 1994). This subject is of considerable interest, because contact angle hysteresis is the direct mechanism which allows droplets to adhere to a solid surface in the presence of flow. Although many previous theories have assumed that $\theta_{A}-\theta_{R}$ is small, we note that the hysteresis may in fact be quite large. As an example, Furmidge (1962) measured the contact angles for water with various surfactants on hydrocarbon surfaces (bees-wax, cellulose acetate). With $\theta_{R}=0^{\circ}$, values of $\theta_{A}$ as high as $45^{\circ}$ were observed, while hysteresis in the range of $20^{\circ}-30^{\circ}$ was observed as $\theta_{R}$ increased to values as high as $87^{\circ}$. Values of $\theta_{A}-\theta_{R}$ in the range of $20^{\circ}-30^{\circ}$ have been routinely reported for gas-liquid systems, while substantially higher values may occur for liquid-liquid systems, (Good \& Koo 1979; Good 1979). 
In the light of this discussion, we identify two classes of problems for equilibrium profiles for droplets on surfaces. The first class (studied by Feng \& Basaran with the additional restrictions $\lambda=0, B_{d}=0$ ) may be stated:

(I) for contact lines at fixed positions determine profiles $\Gamma$ which satisfy (1)-(7) at specified $C a, B_{d}$ and $\lambda$ for given droplet volume $V_{0}=\pi a^{2}$.

The second class of problems may be stated

(II) for contact angles $\theta_{1}$ and $\theta_{2}$ in the range $\theta_{R}<\theta<\theta_{A}$ determine profiles $\Gamma$ which satisfy (1)-(7) at specified $C a, B_{d}$ and $\lambda$ for given droplet volume $V_{0}=\pi a^{2}$.

Experience shows that problems of class I generally possess a single solution for a given $C a, B_{d}$ and $\lambda$. By contrast, the inequality constraint for problems of class II leads to a family of solutions corresponding to different values of $\theta_{1}, \theta_{2}$ within the allowable range. Individual solutions from this class may be determined by specifying one of the angles a priori and solving for $\Delta \theta$. Specifically, in this paper, we seek equilibrium profiles by specifying the contact angle $\theta_{2}=\theta_{A}$ at the trailing contact point, the position at the leading point and the capillary number. The algorithm solves for the equilibrium profile which determines the position of the trailing contact point, the leading contact angle $\theta_{1}$ and hence $\Delta \theta$. As the capillary number is increased, the hysteresis needed to balance the hydrodynamic force increases until the required $\Delta \theta$ reaches the maximum allowed by the constraint, i.e. $\Delta \theta=\theta_{A}-\theta_{R}$. At this limiting $C a$, a single solution exists with $\theta_{1}=\theta_{R}$ and $\theta_{2}=\theta_{A}$; at higher $C a$, no solution exists which satisfies the inequality constraint on contact angles.

It should be noted that for two-dimensional problems, the distinction between the two classes is in the approach, and not in the final results. Thus a collection of solutions from class I may be catalogued in class II by sorting a posteriori according to the contact angles found in the solution. This approach cannot be extended to three dimensions, since the perimeters of the contact line will assume different shapes. Thus the elliptical contact lines assumed by Li \& Pozrikidis (1996) in their class I problem will not coincide with the actual contours for class II problems (Dussan 1987; Furmidge 1962). In the numerical results presented in this paper, we shall consider problems of class II and report our results in this form. Additional results for class I problems are given by Dimitrakopoulos (1996).

\subsection{Boundary integral formulation}

The fundamental solution for the two-dimensional Stokes equations is designated $S_{i j}$ and the associated stress is $T_{i j k}$. These solutions may be written in the form

$$
\begin{gathered}
S_{i j}=-\delta_{i j} \ln r+\frac{\hat{x}_{i} \hat{x}_{j}}{r^{2}}, \\
T_{i j k}=-4 \frac{\hat{x}_{i} \hat{x}_{j} \hat{x}_{k}}{r^{4}},
\end{gathered}
$$

where $\hat{\boldsymbol{x}}=\boldsymbol{x}-\boldsymbol{x}_{0}$ and $r=|\hat{\boldsymbol{x}}|$.

By introducing the fundamental solution and integrating over a volume of fluid bounded by a surface $S$, the velocity at a point $\boldsymbol{x}_{0}$ on the surface is expressed

$$
\boldsymbol{u}\left(\boldsymbol{x}_{0}\right)=-\frac{1}{2 \pi \mu} \int_{S}[\boldsymbol{S} \cdot \boldsymbol{f}-\mu \boldsymbol{T} \cdot \boldsymbol{u} \cdot \boldsymbol{n}] \mathrm{d} S .
$$

The derivation of this integral formula is presented in standard references, see Higdon (1985), Pozrikidis (1992). Here we have chosen the unit normal vector pointing into the volume of fluid.

For a fluid droplet attached to a solid surface, one may model the effect of the solid 


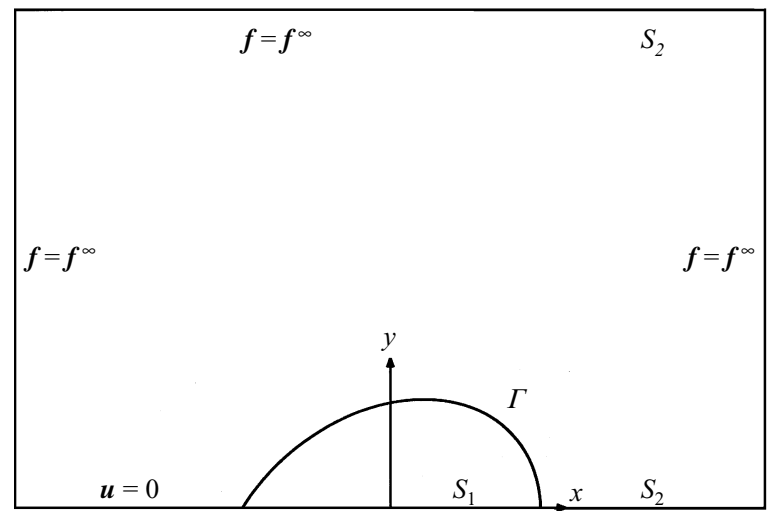

FIGURE 2. Domain geometry for boundary integral solution for viscous flow past droplet attached to a solid boundary.

in two ways. In the first, one employs the Green's function for a half-space bounded by a solid plane (Pozrikidis 1992), and the surface of integration in (10) reduces to that of the fluid-fluid interface. In the second approach, one employs the free-space Green's function (8), and the solid boundary becomes part of the boundary surface $S$. In the numerical solution, the first approach leads to fewer unknowns, while the second approach leads to simpler programming effort and numerical integration. In addition, the second approach is readily extended to solid boundaries of more general shape. Either method is quite effective for the present problem, and we have chosen the second approach with the boundary surfaces as shown in figure 2 . We choose a large rectangular domain occupied by fluid 2 whose outer perimeter is designated $S_{2}$, while the portion of the boundary wall in contact with fluid 1 is designated $S_{1}$, and the interface between the fluids is $\Gamma$. The boundary conditions on the outer surface $S_{2}$ are the no-slip condition on the wall and stress boundary conditions $\boldsymbol{f}=\boldsymbol{f}^{\infty}$ on the fluid portion. In the limit as the surface $S_{2}$ approaches infinity, the solution approaches that for an unbounded fluid. The use of stress boundary conditions on $S_{2}$ is favoured because the limiting behaviour is reached sooner than for velocity boundary conditions.

Following standard procedure, one may write expressions (10) for each fluid and combine the results to achieve an integral formula

$$
\begin{aligned}
\Omega \boldsymbol{u}\left(\boldsymbol{x}_{0}\right)= & -\int_{S_{2}}\left[\boldsymbol{S} \cdot \boldsymbol{f}_{2}-\mu \boldsymbol{T} \cdot \boldsymbol{u}_{2} \cdot \boldsymbol{n}\right] \mathrm{d} S \\
& +\int_{S_{1}}\left[\boldsymbol{s} \cdot \boldsymbol{f}_{1}-\lambda \mu \boldsymbol{T} \cdot \boldsymbol{u}_{1} \cdot \boldsymbol{n}\right] \mathrm{d} S \\
& -\int_{\Gamma}[\boldsymbol{S} \cdot \Delta \boldsymbol{f}-(1-\lambda) \mu \boldsymbol{T} \cdot \boldsymbol{u} \cdot \boldsymbol{n}] \mathrm{d} S
\end{aligned}
$$

which gives the velocity for a point located on the boundary surfaces. The coefficient $\Omega$ takes values $2 \pi \mu(1+\lambda), 2 \pi \lambda \mu$ and $2 \pi \mu$ for points $\boldsymbol{x}_{0}$ on surfaces $\Gamma, S_{1}$ and $S_{2}$ respectively. We have taken the unit normal to point into the volume of fluid 2 and out of the volume of fluid 1 . This yields a consistent definition on the interface $\Gamma$.

For a boundary value problem with known velocity, the integral formula (10) combined with the boundary data yields an integral equation for the unknown surface stresses. For a droplet with interfacial boundary conditions (5), (6), the 
integral formula (11) yields an integral equation for the velocity at the interface and the unknown stresses or velocities on the outer boundaries. In the Newton iteration procedure described below, we shall make use of both of these results.

\subsection{Implementation of Newton iteration}

The boundary integral formulation above provides an efficient method for determining the velocity and stress on an interface of prescribed shape. We now develop an efficient procedure for determining equilibrium profiles. Our basic approach is to adopt a boundary perturbation scheme analogous to that used in asymptotic analyses. In this procedure, we solve the boundary integral equations for a surface of known shape subject to the boundary conditions (5), (6) requiring continuity of velocity and stress at the interface. We then consider arbitrary perturbations of the boundary shape to determine the profile which satisfies the additional constraint imposed by the kinematic condition (7).

Let an interface of known shape be designated $\Gamma_{0}$ and let the positions along this curve be identified as $\boldsymbol{x}_{0}(\xi)$ where $\xi$ is any parametric variable describing the curve. Let positions along a second interface $\Gamma$ be defined by points $\boldsymbol{x}(\xi)$. Define the displacement $d(\xi)$ such that points on $\Gamma$ are related to points on $\Gamma_{0}$ by

$$
\boldsymbol{x}(\xi)=\boldsymbol{x}_{0}(\xi)+d(\xi) \boldsymbol{p}(\xi)
$$

where $\boldsymbol{p}$ is a prescribed vector. (In effect, we will search for new surfaces $\Gamma$ by specifying the direction of displacement $\boldsymbol{p}$, and solving for the magnitude of the displacement $d$.)

Let the unit normal vectors on $\Gamma_{0}$ and $\Gamma$ be defined as $\boldsymbol{n}_{0}$ and $\boldsymbol{n}$ respectively and define $\boldsymbol{n}^{\prime}=\boldsymbol{n}-\boldsymbol{n}_{0}$. The unit tangent vectors $\boldsymbol{t}_{0}, \boldsymbol{t}$ and hence unit normals on the two surfaces may be obtained by differentiating (12). This leads to an expression for $\boldsymbol{n}^{\prime}$

$$
\boldsymbol{n}^{\prime}=-\boldsymbol{t}_{0}\left[\left(\boldsymbol{n}_{0} \cdot \frac{\mathrm{d} \boldsymbol{p}}{\mathrm{d} \xi}\right) d+\left(\boldsymbol{n}_{0} \cdot \boldsymbol{p}\right) \frac{\mathrm{d} d}{\mathrm{~d} \xi}\right]_{\Gamma_{0}} /\left(\frac{\mathrm{d} s}{\mathrm{~d} \xi}\right)_{\Gamma_{0}}+O\left(d^{2}\right)
$$

where $\mathrm{d} s$ is the differential arclength, e.g. $\mathrm{d} s / \mathrm{d} \xi=|\mathrm{d} \boldsymbol{x} / \mathrm{d} \xi|$.

Let the interface $\Gamma$ be considered as a perturbation of the interface $\Gamma_{0}$. The velocity on the interface $\Gamma$ may then be written as a Taylor series about the unperturbed surface $\Gamma_{0}$ with a different expansion for each fluid. (This follows the classic perturbation procedure for a two-phase system.) For the interior and exterior fluids, the velocity on $\Gamma$ becomes

$$
\begin{aligned}
& \left(\boldsymbol{u}_{1}\right)_{\Gamma}=\left(\boldsymbol{u}_{1}\right)_{\Gamma_{0}}+\left(\frac{\partial \boldsymbol{u}_{1}}{\partial n}\right)_{\Gamma_{0}}\left(\boldsymbol{p} \cdot \boldsymbol{n}_{0}\right) d+\left(\frac{\partial \boldsymbol{u}_{1}}{\partial s}\right)_{\Gamma_{0}}\left(\boldsymbol{p} \cdot \boldsymbol{t}_{0}\right) d+O\left(d^{2}\right), \\
& \left(\boldsymbol{u}_{2}\right)_{\Gamma}=\left(\boldsymbol{u}_{2}\right)_{\Gamma_{0}}+\left(\frac{\partial \boldsymbol{u}_{2}}{\partial n}\right)_{\Gamma_{0}}\left(\boldsymbol{p} \cdot \boldsymbol{n}_{0}\right) d+\left(\frac{\partial \boldsymbol{u}_{2}}{\partial s}\right)_{\Gamma_{0}}\left(\boldsymbol{p} \cdot \boldsymbol{t}_{0}\right) d+O\left(d^{2}\right) .
\end{aligned}
$$

All velocities $\boldsymbol{u}$ in (14), (15) represent the solution for a boundary value problem (1)-(6) specified on surface $\Gamma$. Our goal in the perturbation analysis is to reformulate this as a modified boundary value problem on the known surface $\Gamma_{0}$. Toward this end, let $\overline{\boldsymbol{u}}, \overline{\boldsymbol{\sigma}}$ designate the solution of (1)-(6) for a droplet with unperturbed interface $\Gamma_{0}$ with both quantities evaluated on the interface. For small perturbations, we have

$$
\begin{aligned}
& \left(\boldsymbol{u}_{1}\right)_{\Gamma_{0}}=\overline{\boldsymbol{u}}+O(d), \\
& \left(\boldsymbol{u}_{2}\right)_{\Gamma_{0}}=\overline{\boldsymbol{u}}+O(d) .
\end{aligned}
$$


We subtract (14) from (15) and note that the left-hand side is zero from the continuity of $\boldsymbol{u}$ on $\Gamma$. We use (16) and (17) to evaluate the derivative and note that the tangent derivatives cancel owing to the continuity of $\overline{\boldsymbol{u}}$ on $\Gamma_{0}$. The result is

$$
\left(\boldsymbol{u}_{2}-\boldsymbol{u}_{1}\right)_{\Gamma_{0}}+\left(\frac{\partial \overline{\boldsymbol{u}}_{2}}{\partial n}-\frac{\partial \overline{\boldsymbol{u}}_{1}}{\partial n}\right)_{\Gamma_{0}}\left(\boldsymbol{p} \cdot \boldsymbol{n}_{0}\right) d=0 .
$$

In effect, we have transferred the velocity boundary condition (5) for the perturbed solution to the unperturbed boundary $\Gamma_{0}$.

Proceeding in a similar fashion, we may transfer the stress boundary condition (6) to the unperturbed surface. Here we must expand $\sigma$ in a Taylor series, and then take the inner product with $\boldsymbol{n}$ on the perturbed surface $\Gamma$. After some algebra, the result is

$$
\left(\boldsymbol{f}_{2}-\boldsymbol{f}_{1}\right)_{\Gamma_{0}}=-\mathscr{F} d-\left(\overline{\boldsymbol{\sigma}}_{2}-\overline{\boldsymbol{\sigma}}_{1}\right) \cdot \boldsymbol{n}^{\prime}+\gamma(\nabla \cdot \boldsymbol{n}) \boldsymbol{n}+\left(\rho_{2}-\rho_{1}\right)(\boldsymbol{g} \cdot \boldsymbol{x}) \boldsymbol{n}
$$

where

$$
\mathscr{F}=\left(\boldsymbol{p} \cdot \boldsymbol{n}_{0}\right)\left(\frac{\partial \overline{\boldsymbol{\sigma}}_{2}}{\partial n}-\frac{\partial \overline{\boldsymbol{\sigma}}_{1}}{\partial n}\right) \cdot \boldsymbol{n}_{0}+\left(\boldsymbol{p} \cdot \boldsymbol{t}_{0}\right)\left(\frac{\partial \overline{\boldsymbol{\sigma}}_{2}}{\partial s}-\frac{\partial \overline{\boldsymbol{\sigma}}_{1}}{\partial s}\right) \cdot \boldsymbol{n}_{0} .
$$

The curvature term $\gamma(\nabla \cdot \boldsymbol{n}) \boldsymbol{n}$ in (19) may be expressed in terms of the unperturbed value and functions and derivatives of $d$ in an expression analogous to that given for $\boldsymbol{n}^{\prime}$ in (13). The explicit result is given by Dimitrakopoulos (1996).

With (18) and (19), we now have boundary conditions for the velocity and stress for the perturbed boundary value problem expressed as conditions on the unperturbed interface $\Gamma_{0}$. Thus we may choose an arbitrary $d$ and solve for any surface $\Gamma$ by using the same boundary integral equation on $\Gamma_{0}$ changing only the boundary data in (18) and (19). Specifically, with these boundary conditions, the boundary integral formula (11) generalizes to

$$
\begin{aligned}
\Omega_{1} \boldsymbol{u}_{1}\left(\boldsymbol{x}_{0}\right)+\Omega_{2} \boldsymbol{u}_{2}\left(\boldsymbol{x}_{0}\right)= & -\int_{S_{2}}\left[\boldsymbol{S} \cdot \boldsymbol{f}_{2}-\mu \boldsymbol{T} \cdot \boldsymbol{u}_{2} \cdot \boldsymbol{n}\right] \mathrm{d} S \\
& +\int_{S_{1}}\left[\boldsymbol{S} \cdot \boldsymbol{f}_{1}-\lambda \mu \boldsymbol{T} \cdot \boldsymbol{u}_{1} \cdot \boldsymbol{n}\right] \mathrm{d} S \\
& -\int_{\Gamma_{0}}\left[\boldsymbol{S} \cdot\left(\boldsymbol{f}_{2}-\boldsymbol{f}_{1}\right)-\mu \boldsymbol{T} \cdot\left(\boldsymbol{u}_{2}-\lambda \boldsymbol{u}_{1}\right) \cdot \boldsymbol{n}\right] \mathrm{d} S
\end{aligned}
$$

where the coefficient $\Omega_{1}$ is $2 \pi \lambda \mu$ for points $\boldsymbol{x}_{0}$ on $\Gamma_{0}$ and $S_{1}$ and zero for points on $S_{2}$, while $\Omega_{2}$ is $2 \pi \mu$ for points $x_{0}$ on $\Gamma_{0}$ and $S_{2}$ and zero for points on $S_{1}$.

The boundary integral equation (21) presents an efficient approach for studying a large number of trial surfaces $\Gamma$. The discretized form of this equation yields a linear system of algebraic equations $\boldsymbol{A} \boldsymbol{x}=\boldsymbol{b}$ for the unknown surface velocities. The system matrix for these equations is a function only of the unperturbed shape $\Gamma_{0}$, while the perturbation $d$ defining $\Gamma$ affects only the right-hand side. Thus the solution for each successive $\Gamma$ requires no additional numerical quadratures, and negligible computational effort if the equations are solved by Gaussian elimination, and the LU factorization is stored from a previous solution. While this is indeed an efficient approach, we may go one step further. If we leave $d$ unspecified and enforce the kinematic condition (7) on the perturbed surface $\Gamma$, we obtain an additional equation which may be used to solve for $d$. Using (14), we transfer the kinematic condition on $\Gamma$ to the unperturbed surface $\Gamma_{0}$, and find

$$
(\boldsymbol{u})_{\Gamma_{0}} \cdot \boldsymbol{n}_{0}=-\overline{\boldsymbol{u}} \cdot \boldsymbol{n}^{\prime}-\left[\left(\boldsymbol{p} \cdot \boldsymbol{n}_{0}\right)\left(\frac{\partial \overline{\boldsymbol{u}}}{\partial n}\right)+\left(\boldsymbol{p} \cdot \boldsymbol{t}_{0}\right)\left(\frac{\partial \overline{\boldsymbol{u}}}{\partial s}\right)\right] \cdot \boldsymbol{n}_{0} d .
$$


The kinematic condition (22) applies to both $\boldsymbol{u}_{1}$ and $\boldsymbol{u}_{2}$, however specifying either guarantees the other owing to the continuity of velocity (5). The kinematic condition may be used to eliminate one component of the unknown velocity vector at the interface. The unknown displacement $d$ takes its place, leaving the same number of unknowns as in the previous integral equations.

The steps in the Newton iteration for the equilibrium profiles $\Gamma$ are as follows:

Step 1: solve (11) with boundary conditions (5) and (6) to find the velocity $\overline{\boldsymbol{u}}$ on the interface $\Gamma_{0}$.

Step 2: with the velocity $\overline{\boldsymbol{u}}$ known, solve an equation of the form (10) for the surface stresses $\overline{\boldsymbol{f}}_{1}, \overline{\boldsymbol{f}}_{2}$. (The boundary condition (6) gave only the jump $\Delta \boldsymbol{f}$, and not the individual stresses.) This requires a single boundary integral equation solution, because the stresses are related by $\overline{\boldsymbol{f}}_{2}=\overline{\boldsymbol{f}}_{1}+\Delta \boldsymbol{f}$ with $\Delta \boldsymbol{f}$ known. $\dagger$

Step 3: evaluate the derivatives $\partial \overline{\boldsymbol{u}} / \partial n, \partial \overline{\boldsymbol{u}} / \partial s, \partial \overline{\boldsymbol{\sigma}} / \partial n \cdot \boldsymbol{n}_{0}, \partial \overline{\boldsymbol{\sigma}} / \partial s \cdot \boldsymbol{n}_{0}$ required for boundary data in (18), (19) and (22).

Step 4: solve (21) with boundary conditions (18), (19) and (22) and with constant drop volume, to determine the displacement $d$ as described above.

This procedure requires three boundary integral solutions per iteration, however the kernels $\boldsymbol{S}$ and $\boldsymbol{T}$ are identical in each case and numerical quadratures need be executed only once. The derivatives $\partial / \partial s$ in Step 3 require differentiation of known quantities along the surface. The derivatives $\partial / \partial n$ may be evaluated indirectly given the values of $\boldsymbol{\sigma}$ and the equations $\nabla \cdot \boldsymbol{u}=0, \boldsymbol{\nabla} \cdot \boldsymbol{\sigma}=0$. All numerical derivatives are evaluated using the so-called collocation derivative, that is using analytical differentiation of the high-order polynomials which comprise the basis functions for the spectral element expansions. See Muldowney \& Higdon (1995) or Canuto et al. $(1988, \S 2.3,2.4)$ for additional details.

A comment may be warranted concerning the constant-volume constraint in Step 4. While the solution of the boundary integral equation yields a velocity field which is divergence free, the boundary perturbation represented by $d$ admits a broad class of surfaces which may include different volumes of fluid. We add the volume constraint to assure that the equilibrium profile corresponds to the specified fluid volume $V_{0}$.

In the Newton iteration procedure, we assume that the direction of the search vector $\boldsymbol{p}$ is specified a priori. For freely suspended droplets, a reasonable choice is simply the unit normal vector $\boldsymbol{n}$. For droplets attached to a plane wall, we need to modify this choice, because a displacement in the normal direction would move the end points of the interface away from the wall. We need to define $\boldsymbol{p}$ such that the end points are free to move along the wall, but remain in the plane $y=0$. As an obvious choice, we define $\boldsymbol{p}(\xi)$ parallel to the wall at the end points and smoothly interpolate to $\boldsymbol{n}$ as $\boldsymbol{x}(\xi)$ moves away from the wall. This choice admits a class of profiles $\Gamma$ with arbitrary contact angles and arbitrary contact positions along the wall.

\subsection{Numerical methods}

The numerical solution of the boundary integral equation of $\S 2.2$ is achieved through the spectral boundary element method (Occhialini et al. 1992; Muldowney \& Higdon 1995). Briefly, each boundary is divided into a small number of surface elements which are parameterized by a variable $\xi$ on the interval $[-1,1]$. The geometry and physical

$\dagger$ As an alternative to Step 2, the surface stress may be evaluated directly as an integral of $\Delta \boldsymbol{f}$ and $\boldsymbol{u}$ over the boundary surfaces. The kernels required for this integration are the two dimensional versions of $\boldsymbol{T}$ and $\boldsymbol{Q}$ as given in Muldowney \& Higdon (1995). These kernels require additional time for numerical quadratures, and the preferred choice will be dictated by a balance between quadrature effort and matrix inversion time. 
variables are discretized using Lagrangian interpolation with the $N_{B}$ basis points chosen as the zeros of the $N_{B}$-order Legendre polynomial. This is equivalent to an orthogonal polynomial expansion and yields the spectral convergence associated with such expansions. The discretizations are substituted into the appropriate boundary integrals and quadratures evaluated using adaptive Gaussian quadrature. Further details are given in the aforementioned references.

The spectral element method as implemented here employs basis points in the interior of the element. Owing to this choice, additional constraints are required at the ends of the elements to enforce continuity of position and slope on the interface. As an alternative, different basis sets may be used to enforce these constraints implicitly. Gauss-Lobatto points maintain continuity of position, while basis points derived from Jacobi polynomials are required for continuity of both position and slope. The use of these alternative basis sets adds to the complexity of the programming effort, and we did not pursue this strategy in the present work. The boundary conditions on the contact angle and/or contact line position must be added as further constraints for the linear system. The discretized system of equations is then solved using a least-squares algorithm from the LAPACK software library.

\section{Results}

In this section, we present the results of a comprehensive series of computations based on the methods described in $\S 2$. Numerous tests were conducted to verify the reliability and robustness of these algorithms. The performance of the basic spectral element algorithm has been documented previously (Occhialini et al. 1992; Muldowney \& Higdon 1995). Additional tests were performed on the present implementation as described by Dimitrakopoulos (1996). Various discretizations were employed with $N_{B}=4$ to 18 demonstrating exponential convergence comparable to that in previous applications of this algorithm. Several additional tests were performed to verify the performance of the Newton iteration procedure. Two classes of tests were conducted, for freely suspended droplets and for droplets attached to a plane wall. For the freely suspended droplets, equilibrium profiles were compared with results based on time-dependent computations and with the analytical solution of Richardson $(1968,1973)$ for the special case $\lambda=0$. For the attached droplets, detailed comparisons were made with the results of Feng \& Basaran (their figures 6 and 7) for the case $R e=0$. All computations were in excellent agreement with earlier results. See Dimitrakopoulos (1996) for explicit comparisons. In addition to these checks, the numerical results were found to be in excellent agreement with asymptotic theories as described below. For the majority of results presented in this section, the droplet interface was divided into $N_{E}=6$ elements with an $N_{B}=10$ spectral expansion on each element. For certain cases with small angles (e.g. $\theta_{A}=10^{\circ}$ ), a higher-order basis or more elements were employed. In all cases, numerical convergence was verified by varying the order of the spectral expansion. The data for the critical $\mathrm{Ca}$ versus $\Delta \theta$ were computed with a maximum relative error of $1 \%$ in nearly all cases. For the highest deformations, the relative error in a few runs at small $\theta_{A}$ reached $3 \%$. These cases corresponded to large deformation where the receding contact angle was of order $5^{\circ}$. In the limit of small contact angles, lubrication theory (Johnson 1981) predicts a singularity in the pressure near the contact lines making accurate numerical computations more challenging.

The Newton iteration algorithm introduced in this paper is a robust procedure which may prove useful in other boundary integral applications. To demonstrate the 


$\begin{array}{crccc}\text { Iteration } & d_{\max } & u_{\max } & d_{\max } & u_{\max } \\ 1 & 2.8 \times 10^{-1} & 2.1 \times 10^{-1} & 2.4 \times 10^{-1} & 2.7 \times 10^{-1} \\ 2 & 6.8 \times 10^{-2} & 2.5 \times 10^{-2} & 1.5 \times 10^{-1} & 1.9 \times 10^{-1} \\ 3 & 3.0 \times 10^{-3} & 6.6 \times 10^{-4} & 6.2 \times 10^{-2} & 1.2 \times 10^{-1} \\ 4 & -3.6 \times 10^{-4} & 1.3 \times 10^{-5} & 6.5 \times 10^{-2} & 4.8 \times 10^{-2} \\ 5 & 2.1 \times 10^{-6} & 1.7 \times 10^{-6} & 2.4 \times 10^{-2} & 7.7 \times 10^{-3} \\ 6 & -1.5 \times 10^{-9} & 1.7 \times 10^{-6} & 4.9 \times 10^{-4} & 6.7 \times 10^{-4} \\ 7 & & & 1.6 \times 10^{-5} & 2.1 \times 10^{-5} \\ 8 & & & -4.5 \times 10^{-7} & 1.5 \times 10^{-6} \\ 9 & & & -2.2 \times 10^{-8} & 4.7 \times 10^{-7} \\ 10 & & & -1.5 \times 10^{-9} & 4.3 \times 10^{-7}\end{array}$

TABLE 1. Convergence of Newton iteration for free surface flows: maximum displacement $d$ and maximum normal velocity evaluated over droplet surface. Columns 2 and 3 are for a droplet freely suspended in extensional flow; columns 4 and 5 are for a droplet attached to a wall. Interface profiles are shown in figure 3.

potential of this method and its range of convergence, we consider two examples: a freely suspended droplet in an extensional flow and a droplet attached to a wall in a shear flow. In each case, the initial profile is that for a quiescent fluid. Figure 3 illustrates the convergence of the droplet profiles, while table 1 shows the convergence of the normal velocity and the displacement $d$. These examples were chosen to show that the method converges rapidly even for large differences between the initial guess and the final shape. In the initial iterations, the method does not show the quadratic convergence $d_{n+1} \sim d_{n}^{2}$ characteristic of Newton's method, because the initial guess is far from the equilibrium solution and $d$ is not sufficiently small. In later steps, when the displacement is reduced below $10^{-3}$ (relative to drop radius), the convergence is quite rapid, but still fails to achieve a quadratic rate. The analytical derivations leading to the boundary integral equation (21) are accurate to $O\left(d^{2}\right)$, and thus should produce a quadratic convergence rate. The failure to achieve the full quadratic convergence for small $d$ is associated with discretization error in the boundary integral solution and with errors associated with numerical differentiation in Step 3 of the algorithm. In the boundary integral formulation, the continuity of velocity, surface stress and surface geometry is not enforced by the basis functions, and these conditions must be added as explicit constraints. This yields an overdetermined system of equations which is solved using a least-squares algorithm. The residuals in this solution scale as a small number multiplied by $d$. When $d$ is large, these residuals are smaller than $d^{2}$ and quadratic convergence is achieved. As convergence continues, $d$ is reduced and the small residuals from the linear system become the dominant error term. In this case, the rate of convergence becomes linear in $d$. To eliminate this problem, one would require basis functions with continuity of the function and first derivative at element boundaries. The appropriate basis functions are derived from Jacobi polynomials, but the use of this basis and the implicit coupling across element borders would significantly complicate the programming effort for the algorithm.

Despite the failure to achieve quadratic convergence, the displacement and normal velocity in all cases show exponential convergence $\mathrm{e}^{-c n}$ with the number of iterations, yielding performance far superior to previous techniques employed with the boundary integral method. After the initial iterations where $d$ is large, the data of table 1 show a reduction in $d$ of 4 to 5 orders of magnitude in just 3 iterations. As noted, the examples shown here were chosen to illustrate the large range of convergence. 

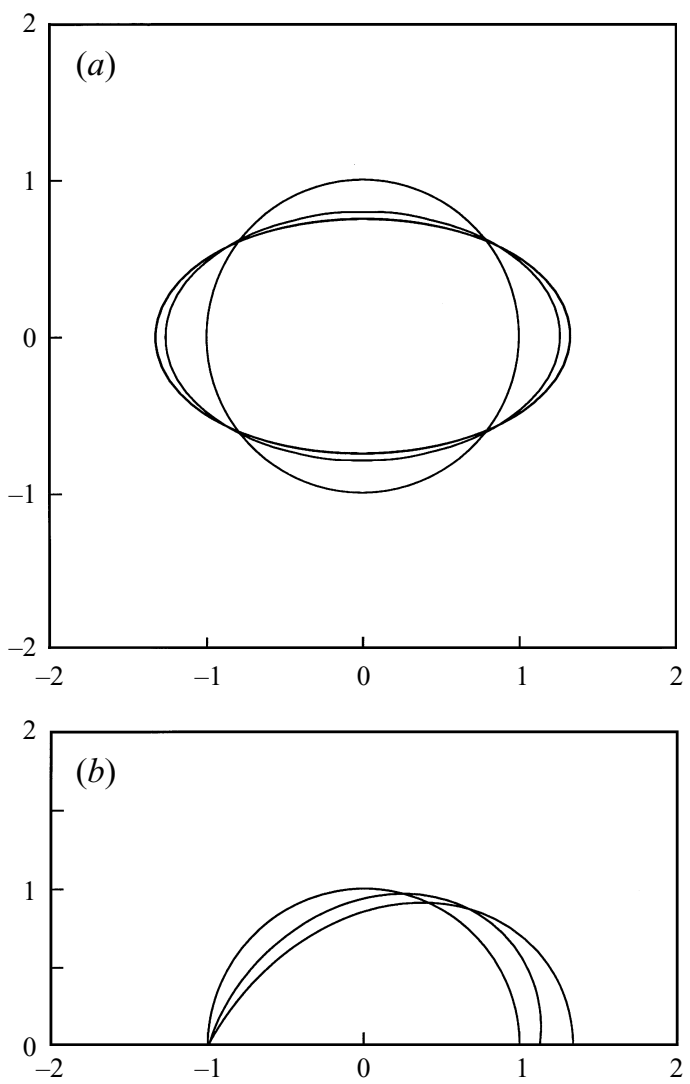

Figure 3. Convergence test for the Newton iteration scheme. (a) Droplet in extensional flow, $\lambda=1$, $C a=0.125$ : initial shape, 1st and final iterations; $(b)$ droplet on solid wall, $\lambda=1, C a=0.05$, $B_{d}=0, \theta_{A}=90^{\circ}$ : initial shape, 2nd and final iterations.

Additional convergence tests with closer initial approximations show the same high convergence rate starting from the first iteration. For parametric studies, it is most effective to choose initial guesses based on nearby solutions such that a small number of iterations yields an accurate solution. This is consistent with common practice in finite element applications of Newton's method.

\subsection{Drop displacement in the absence of gravity, $B_{d}=0$}

We begin our investigation of drop displacement from a rigid wall by considering shear flows in the absence of gravitational forces $\left(B_{d}=0\right)$. Figure 4 shows the streamlines for four equilibrium profiles in the case of droplets with viscosity ratios $\lambda=1$ and $\lambda=10$ for capillary numbers $C a=0.02$ and $C a=0.08$. The advancing contact angle is $\theta_{A}=90^{\circ}$ in each case, while the difference $\theta_{A}-\theta_{R}$ required for equilibrium at each $\mathrm{Ca}$ may be determined from figure 6 below. Several noteworthy features are apparent from these figures. First, in comparing the outer flows at equal $\mathrm{Ca}$, we see that there is very little change in the velocity field even for this large change in viscosity ratio. Based on the spacing of the streamlines (at equal increments of stream function), we observe only a modest reduction in velocity above the $\lambda=10$ droplet. On the other hand, the velocity field inside the droplets has the same pattern, but its magnitude is greatly reduced for the more-viscous droplets. (The stream 

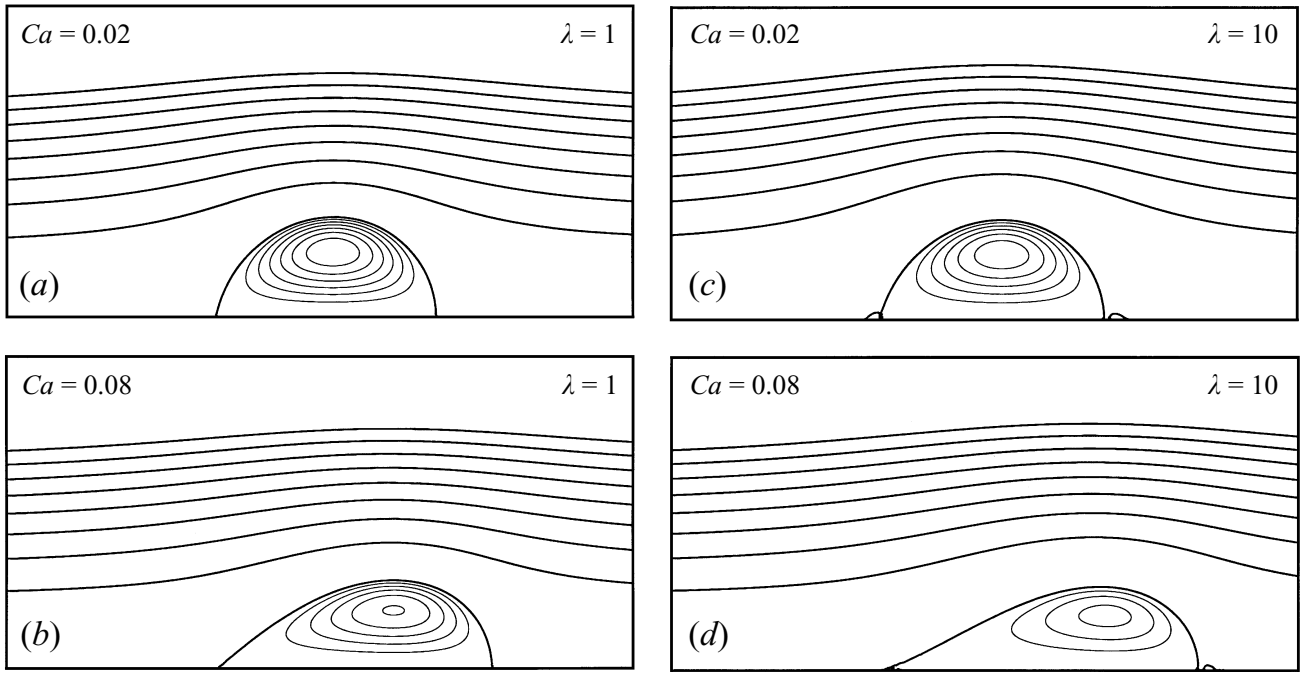

FIGURE 4. Streamlines for shear flow past viscous droplets attached to a solid wall with $B_{d}=0$. All contours are plotted at equal increments of stream function in the exterior flows; contours in the interiors are plotted at spacings $1 / 25$ and $1 / 125$ of the exterior values for the $\lambda=1$ and $\lambda=10$ droplets respectively.

function contours in the interior are plotted at intervals of $1 / 25$ and $1 / 125$ of the external intervals for the $\lambda=1$ and $\lambda=10$ drops respectively.) The more-viscous droplet shows the formation of corner eddies at the contact lines; however these eddies are extremely weak and have little effect on the overall dynamics.

It should be emphasized that the streamlines shown in figure 4 (and in figure 9 below) were computed using a low-order contour algorithm on a rectangular mesh. These streamlines are calculated in a post-processing operation, and the resolution of the streamlines is not representative of the precision of the boundary integral solutions. In particular, the poorly resolved corner eddies for $\lambda=10$ in figure $4(c, d)$ (and in figure $9 c, d$ below) do not reflect the shape of the droplet interface. The droplet interface as calculated in the spectral boundary element algorithm is extremely smooth with continuous slope as shown in figure 5 and figure 10 below.

Comparing the low- $\mathrm{Ca}$ results with the higher- $\mathrm{Ca}$ cases in figure 4, we observe that the trailing contact line has been displaced further downstream as the flow rate has increased. This trend can be seen more clearly by superimposing the equilibrium contours at different flow rates (or $C a$ ) on the same plot. Figure $5(a-c)$ shows the equilibrium contours for three different viscosities $(\lambda=0,1,10)$ all with $\theta_{A}=90^{\circ}$. With each successive contour, the contact angle hysteresis $\theta_{A}-\theta_{R}$ is increased allowing a larger flow rate before the critical $\mathrm{Ca}$ is reached. Each of the droplets in figure $5(a-c)$ has an identical volume of fluid. Earlier in figure 1 we had placed the origin of the coordinate system at the centre of droplet, however in figure 5 all contours are drawn with the upstream contact line fixed, while the movement of the downstream contact line illustrates the increase in the overall length of the droplet. For a smooth wall in a homogeneous shear flow, all positions on the wall are equally favoured, and adjustments to new equilibrium profiles might be reached by movement of either or both of the contact lines. Thus horizontal translations are arbitrary, and we have aligned the leading edges for purposes of clear illustration.

The most distinctive feature of the profiles in figure $5(a-c)$ is that the shapes are 

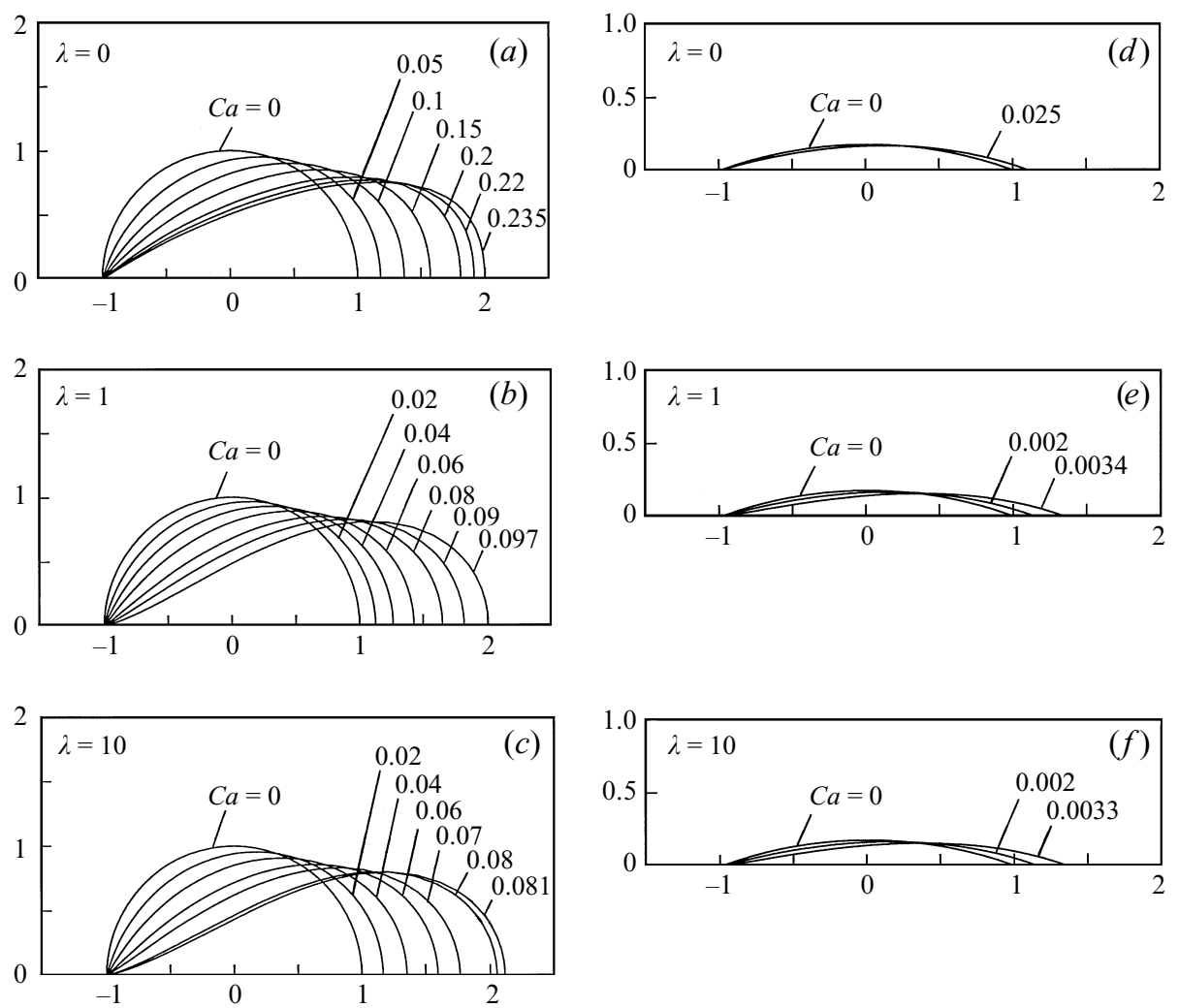

FIGURE 5. Equilibrium profiles for droplets in shear flows with $B_{d}=0$ for large contact angles $\theta_{A}=90^{\circ}(a-c)$ and for small contact angles $\theta_{A}=20^{\circ}(d-f)$.

remarkably similar even as the droplet viscosity varies from $\lambda=0$ to $\lambda=10$. This trend is also seen in the profiles at small contact angles shown in figure $5(d-f)$. One might infer from these figures that the critical capillary number for drop displacement is very little affected by droplet viscosity. This inference would be in serious error as the next figure will show.

Figure $6(a-c)$ show the critical $C a$ as a function of hysteresis $\theta_{A}-\theta_{R}$ for several values of $\theta_{A}$ and for the three viscosity ratios viewed in figure 5 . For each point on a given curve, we specify the value of $\theta_{A}$ and the capillary number, and then solve for the equilibrium profile which determines the value $\theta_{R}$ and hence $\Delta \theta$. The specified $\mathrm{Ca}$ then represents the yield condition for that value of $\theta_{A}$ and $\theta_{A}-\theta_{R}$. For any $\mathrm{Ca}$ below the critical value (e.g. for any lower shear rate), there exists a family of equilibrium solutions with contact angles in the range $\theta_{R} \leqslant \theta_{1}, \theta_{2} \leqslant \theta_{A}$. The existence of a family of solutions at lower $C a$ is a consequence of the inequality constraint on contact angles. The critical $\mathrm{Ca}$ represents the limit for which this family reduces to a single solution with $\theta_{1}=\theta_{R}$ and $\theta_{2}=\theta_{A}$. The terminal points at the end of each curve represent the largest $\Delta \theta$ for which accurate numerical calculations could be executed. (The criteria for an accurate numerical solution include convergence of the Newton iteration, consistency with increasing order of $N_{B}$ and an error tolerance for the maximum $\boldsymbol{u} \cdot \boldsymbol{n} \neq 0$ on the interface.) It appears that the true terminal point based on physical grounds corresponds to a value $\Delta \theta \rightarrow \theta_{A}$, for which $\theta_{R} \rightarrow 0$ and the interface becomes tangent to the boundary wall at the upstream contact point. This 
limit produces a singularity in the pressure (Johnson 1981) and an infinite sequence of Moffatt eddies at the contact point, making numerical computations impractical.

The curves of figure 6 reveal interesting behaviour for the critical $\mathrm{Ca}$ at different viscosity ratios. For the viscous droplets $(\lambda=1, \lambda=10)$ and a given hysteresis $\theta_{A}-\theta_{R}$, increasing the contact angle $\theta_{A}$ increases the critical shear rate or $C a$. For an inviscid droplet $(\lambda=0)$, increasing $\theta_{A}$ decreases the critical shear rate. This phenomenon is much more pronounced at the smaller contact angles $\theta_{A}$. The explanation for this curious phenomenon is quite simple when one considers the basic physics involved. For viscous droplets with small contact angles, the primary hydrodynamic force is the shear stress which is very close to $\tau_{\infty} \equiv \mu G$ independent of the internal viscosity. As $\theta_{A}$ increases, the height of the drop increases, while its length decreases. The hydrodynamic force scales as $\ell \tau_{\infty}$, hence a reduced length requires a higher shear rate to overcome the surface force $\gamma \theta_{A} \Delta \theta$ which is independent of length. (See the Appendix for an asymptotic analysis.) By contrast, the shear stress on the inviscid droplet is identically zero, and the hydrodynamic force is due to the pressure field. The pressure force on the inviscid droplet is insensitive to the length and scales with the droplet height. Increasing the contact angle $\theta_{A}$ increases the height and pressure force, hence a lower flow rate is sufficient to displace the droplet. Based on these phenomena one should be cautious in extrapolating results for a given viscosity ratio to droplets of different viscosities, e.g. in comparing liquid droplets and gas bubbles.

The viscosity behaviour seen in figure 6 may be surprising given that previous authors (Dussan 1987) have found that the critical capillary number is independent of viscosity ratio to leading order. This conclusion is correct, but needs to be interpreted within the limits of the theory. In the Appendix, we present the simple two-dimensional analogue of Dussan's lubrication theory. The critical capillary number is given by (A4):

$$
C a_{\text {asym }}=\left(\frac{2}{27 \pi}\right)^{1 / 2} \theta_{A}^{3 / 2} \Delta \theta .
$$

For comparison, Dussan's theory for three-dimensional droplets predicts

$$
C a_{\text {asym }} \sim \theta_{A}^{4 / 3} \Delta \theta .
$$

Each of these results is indeed independent of $\lambda$. The problem lies in the fact that $\lambda \rightarrow 0$ is a singular limit. The shear stress on the drop scales as $\tau_{\infty}$ for any finite $\lambda$, but is identically zero for $\lambda=0$. The pressure force is much smaller than the shear stress for finite $\lambda$, however the pressure force becomes the dominant force for infinitesimal $\lambda$ where the lubrication theory is no longer valid. The critical capillary number undergoes significant changes in scaling when $\lambda$ is very small (see (A 12) in the Appendix.) This reaffirms the fact that one should be extremely cautious in applying lubrication arguments in cases where $\lambda \ll 1$, e.g. for gas bubbles in liquids.

Given the results of the asymptotic lubrication theory for viscous fluids, one would expect the $\mathrm{Ca}$ dependence on viscosity ratio to lessen for small contact angles where this theory is valid. To examine this proposition, we plot the curves for several viscosity ratios on a single graph in figure 7 . Here the numerical results are consistent with the lubrication theory for small angles with the $\lambda$ dependence disappearing as $\theta_{A}$ takes on smaller values. On the other hand, at larger $\theta_{A}$, the very viscous fluids $(\lambda=10, \lambda=100)$ show significant departure from the $\lambda=1$ results. Clearly, the effects of viscosity ratio should not be discounted for viscous fluids with $\lambda \geqslant 1$ and projections based on $\lambda=1$ may give only qualitative predictions for more-viscous 

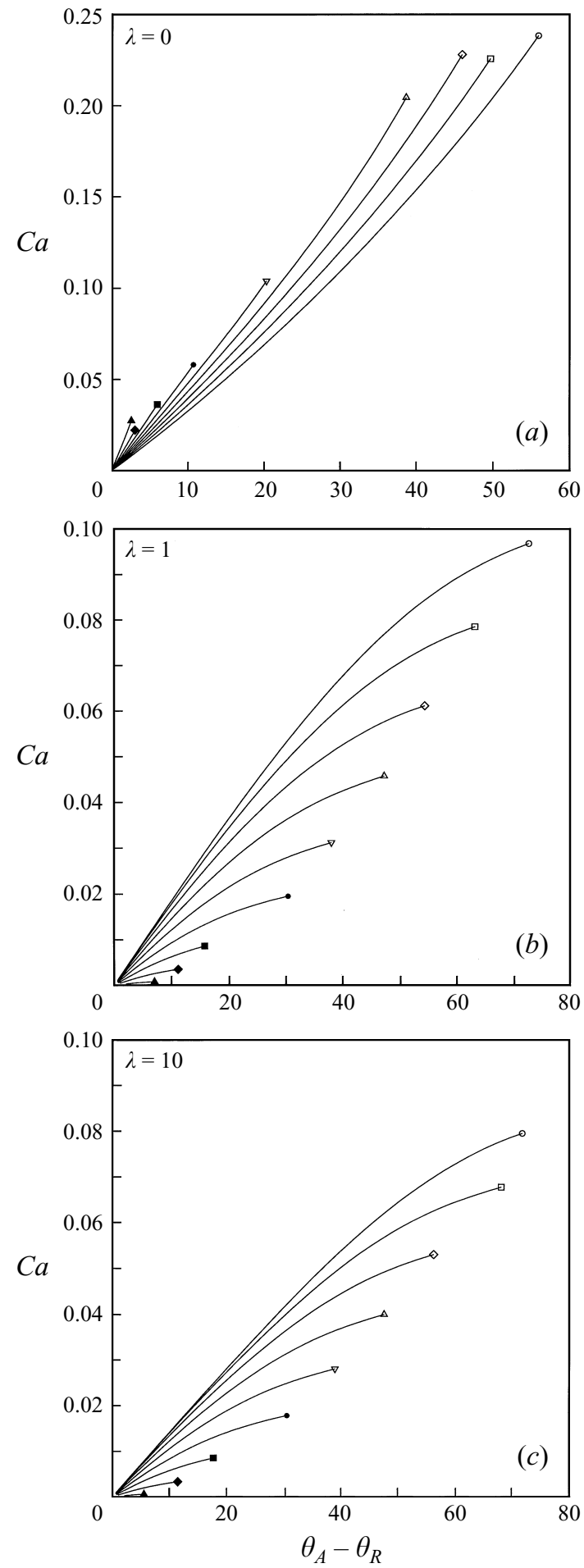

FIgURE 6. Critical capillary number versus hysteresis $\theta_{A}-\theta_{R}$ for $B_{d}=0$ and viscosity ratios $\lambda$ as noted. Contact angles $\theta_{A}: \boldsymbol{\Lambda}, 10^{\circ} ; \diamond, 20^{\circ} ; \mathbf{\square}, 30^{\circ} ; \bullet, 40^{\circ} ; \nabla, 50^{\circ} ; \triangle, 60^{\circ} ; \diamond, 70^{\circ} ; \square, 80^{\circ} ; \circ, 90^{\circ}$. 


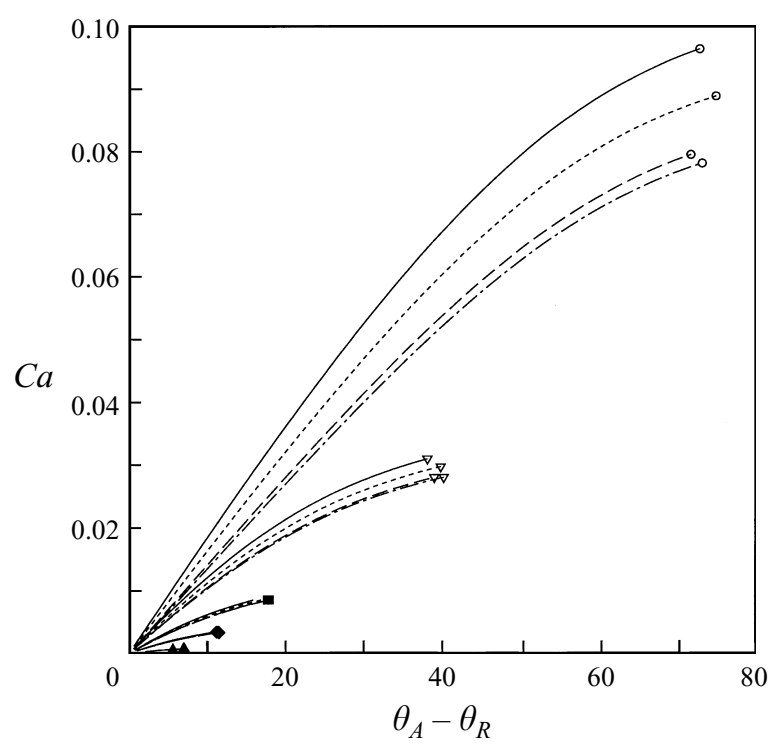

FIGURE 7. Critical capillary number as a function of contact angle hysteresis $\theta_{A}-\theta_{R}$ for $B_{d}=0$. Contact angles $\theta_{A}: \boldsymbol{\Delta}, 10^{\circ} ; \bullet, 20^{\circ} ; \mathbf{\square}, 30^{\circ} ; \nabla, 50^{\circ} ; \circ, 90^{\circ}$; viscosity ratio $\lambda:-, 1 ;-\cdots, 2$; ,$--- 10 ;-\cdot-, 100$.

fluids. Note that the inviscid droplet results are not shown on figure 7; these curves would appear far above and to the left of the plotted curves.

To assess the quantitative accuracy of the asymptotic theory for critical $\mathrm{Ca}$, we plot the ratio of the computed numerical value to the asymptotic prediction in figure 8 . Note that the horizontal axis has been scaled with $\theta_{A}$ yielding the variable $\Delta \theta / \theta_{A}$. The first conclusion is that the quantitative predictions are rather poor, even for relatively small contact angles. In fact, the range of validity is even smaller than this figure might indicate because of the scaling of the abscissa. Dussan's theory as well as our two-dimensional analogue requires not only $\theta_{A} \ll 1$, but also $\Delta \theta \ll \theta_{A}$. Thus the theory is valid only for vanishingly small values of the contact angle hysteresis, i.e. only within a very small region in the upper left corner of figure 8 . Even in the limit as $\Delta \theta \rightarrow 0$, the predictions shown on figure 8 for finite $\theta_{A}$ are not that accurate, as indicated by the intercepts of the curves with the ordinate axis. Despite its limited range, the lubrication approximation does provide a useful check for our numerical computations. Dimitrakopoulos (1996) has extrapolated the numerical results for small $\theta_{A}$ and shown excellent agreement with the asymptotic prediction (A 4).

One might attempt to improve upon the asymptotic theory by relaxing the restriction $\Delta \theta \ll \theta_{A}$. As noted by Dussan, this is quite difficult in the three-dimensional case, involving the solution of a nonlinear partial differential equation for the interface shape. Moreover, the inherent limits of the lubrication approximation make such a computation of questionable value. In the two-dimensional case, we have calculated the next term in the asymptotic expansion for small $\Delta \theta / \theta_{A}=\varepsilon \ll 1$. (The details are omitted but follow the approach of Johnson 1981.) This requires the solution for the first-order perturbation of the droplet profile and yields a correction factor $\left(1-\frac{3}{4} \varepsilon\right)$ which is plotted as the dashed line in figure 8 . We see that this correction yields little improvement in overall accuracy, because it ignores a correction factor $\left(1+O\left(\theta_{A}, \Delta \theta\right)\right)$ associated with perturbations in the outer flow. The perturbation calculation does 


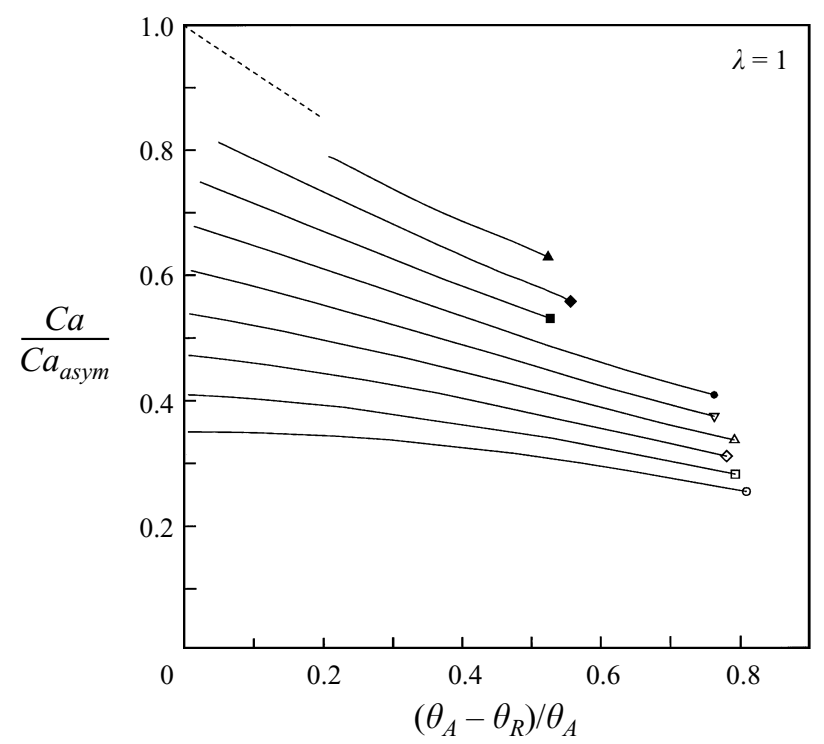

FiguRE 8. Ratio of critical $C a$ to asymptotic predictions based on (A 4) with $B_{d}=0$. Dashed line is asympotic theory with second-order correction for $\Delta \theta$. Contact angles $\theta_{A}: \boldsymbol{\Delta}, 10^{\circ} ; \bullet 20^{\circ} ; \mathbf{\square}, 30^{\circ}$; $\bullet, 40^{\circ} ; \nabla, 50^{\circ} ; \triangle, 60^{\circ} ; \diamond, 70^{\circ} ; \square, 80^{\circ} ; \circ 90^{\circ}$.

yield one interesting result, as it predicts a first-order disturbance profile independent of $\lambda$. This helps explain the similarity of equilibrium profiles shown in figure 5 .

Despite the lack of a higher-order analytical theory for the critical $\mathrm{Ca}$, it may prove useful in some applications to present an empirical equation which correlates the data of figure 6. For the viscous droplets, we may start with equation (A 2), which represents the balance of viscous and interfacial forces. The interfacial force $\gamma\left(\cos \theta_{R}-\cos \theta_{A}\right)$ is an exact result valid for all contact angles. For the viscous force, we may replace the asymptotic result $3 \ell \tau_{\infty}$ with an expansion of the form $3 \ell \tau_{\infty}\left(1+b_{1} \theta_{A}+b_{2} \Delta \theta / \theta_{A}\right)$ where the coefficients $b_{1}$ and $b_{2}$ are to be determined empirically. This approach yields a critical capillary number

$$
C a=\left(\frac{2}{27 \pi}\right)^{1 / 2} \frac{\left(\cos \theta_{R}-\cos \theta_{A}\right) \theta_{A}^{1 / 2}}{1+b_{1} \theta_{A}+b_{2}\left(\Delta \theta / \theta_{A}\right)} .
$$

The expression (23) reduces to the exact asymptotic result (A4) in the limit as $\theta_{A}$ and $\Delta \theta / \theta_{A}$ approach zero. Utilizing (23) and employing a least-squares fit to the data in figure $6(b, c)$ the coefficients $b_{1}$ and $b_{2}$ take values

$$
\left.\begin{array}{rll}
\lambda=1: & b_{1}=0.509, & b_{2}=0.161 \\
\lambda=10: & b_{1}=0.823, & b_{2}=0.065 .
\end{array}\right\}
$$

With these values (23) predicts the critical $C a$ with a maximum relative error of $1.5 \%$ for $\lambda=1$ and $5 \%$ for $\lambda=10$.

Turning to the zero-viscosity results of figure $6(a)$, we note that there is no simple asymptotic theory for inviscid droplets with $\lambda=0$; however, a scaling analysis (A 11) predicts $C a \sim \theta_{A}^{-1 / 2} \Delta \theta$. Following the procedure described above, we may utilize this result, employing the exact expression for the interfacial force and a linearized correction for the hydrodynamic force. We define $\bar{\theta}=\left(\theta_{A}+\theta_{R}\right) / 2$ and employ this quantity in the scaling in place of $\theta_{A}$. This leads to improved results with a correlation 
for the critical $\mathrm{Ca}$ in the form

$$
C a=\frac{\left(\cos \theta_{R}-\cos \theta_{A}\right) \bar{\theta}^{-3 / 2}}{b_{0}+b_{1} \theta_{A}+b_{2}\left(\Delta \theta / \theta_{A}\right)} .
$$

A least-squares fit to the data of figure $6(a)$ determines the coefficients:

$$
\lambda=0: \quad b_{0}=4.515, \quad b_{1}=-1.032, \quad b_{2}=0.299 .
$$

With these values, (25) predicts the critical $C a$ for $\lambda=0$ with a maximum relative error of $4 \%$.

\subsection{Drop displacement with gravitational effects}

Having considered the major phenomena affecting droplet displacement at zero Bond number, we now turn our attention to the effects of gravity. We consider only the case of a vertical gravity vector with increasing Bond number tending to increase the spread of the droplet over the surface. We begin with the streamline patterns in figure 9, which may be compared with the analogous results for $B_{d}=0$ from figure 4 . As before, we see little change in the external flow for $\lambda=1$ and $\lambda=10$, however the interior circulation is much weaker for the more-viscous droplets. We emphasize that the small unresolved corner eddies for $\lambda=10$ are a consequence of the low-order streamline algorithm. The actual droplet interfaces are computed to high precision and show smooth contours as shown in figure 10 below. All profiles for $B_{d}=1$ have greater length than their counterparts for $B_{d}=0$ with significant consequences for the critical displacement conditions. For viscous droplets with equal $\mathrm{Ca}$, these profiles show that $B_{d}=1$ droplets require a greater contact angle hysteresis to maintain their position on the substrate than the zero gravity case. Finally, the figures for $B_{d}=5$ illustrate the asymptotic limit for a very long droplet with nearly parallel streamlines over the bulk of the droplet volume. At this Bond number droplets can withstand only a very small deformation before being displaced from the surface.

Continuing our discussion of gravity effects, we show the equilibrium profiles for $B_{d}=1$ and a range of $C a$ and viscosity ratios in figure 10 . These profiles are qualitatively similar to their counterparts in the $B_{d}=0$ case. As the capillary number is increased, the droplets show a larger change in $\theta_{A}-\theta_{R}$ and a greater extension over the solid boundary. The inviscid droplets show considerably less extension than the viscous droplets under the same conditions. Figure 11 shows the critical capillary number as a function of $\theta_{A}-\theta_{R}$ for several viscosity ratios and contact angles $\theta_{A}$. Note that the critical $\mathrm{Ca}$ for the viscous droplets is in all cases lower for these figures than for the respective cases at $B_{d}=0$. The physical explanation is similar to that discussed earlier. The gravitational force tends to spread the droplet over the plane, increasing the length for a given volume of fluid. The increased length increases the viscous force which scales as $\ell \tau_{\infty}$. With increasing length, the droplet yields its position at smaller shear rates and hence gives a lower critical $\mathrm{Ca}$. As before, decreasing the contact angle adds to this effect with a further increase in length and decrease in $C a$. The droplets for $B_{d}=5$ show the smallest yield conditions owing to their extreme length. These trends are all in qualitative agreement with the asymptotic predictions of lubrication theory as given in the Appendix (A 18), (A 19). We have extrapolated the numerical results for small $\theta_{A}, \Delta \theta$ and found excellent agreement with the asymptotic predictions.

As with the zero gravity case, the inviscid droplets exhibit a behaviour opposite to that of the viscous droplets. The influence of the pressure force scales with droplet height yielding a lower $\mathrm{Ca}$ as the height is increased. This explains the increasing 

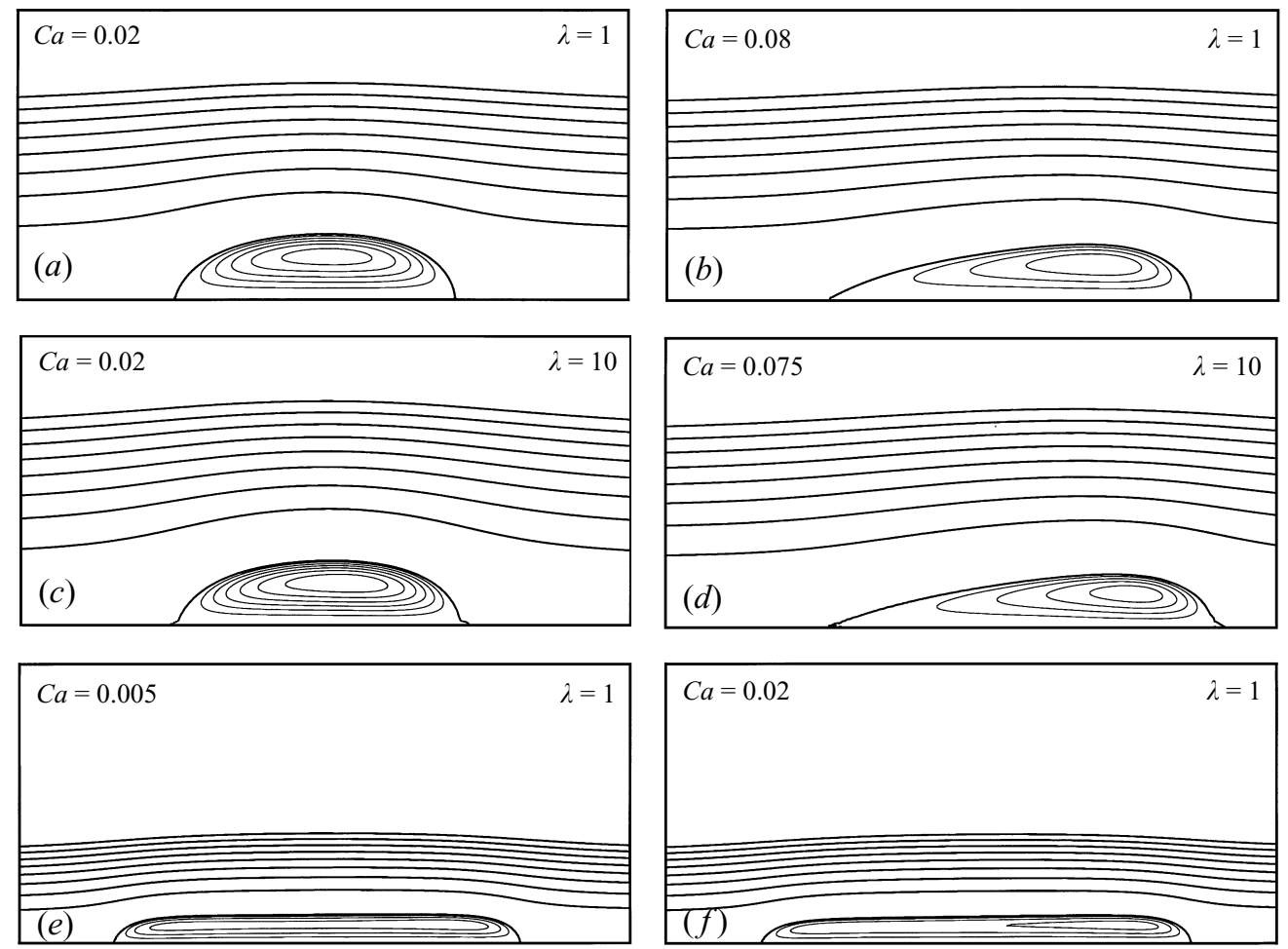

FIGURE 9. Streamlines for droplets in shear flow with gravity effects: $(a-d) B_{d}=1,(e, f) B_{d}=5$. Contours are plotted at equal increments of stream function in the exterior flows; contours in the interiors are plotted at spacings $1 / 50$ and $1 / 500$ of the exterior values for the $\lambda=1$ and $\lambda=10$ droplets respectively.

$\mathrm{Ca}$ as the contact angle $\theta_{A}$ decreases. An interesting summary of these phenomena is presented in figure 12 where we gather results for viscosities $\lambda=0$ and $\lambda=1$ and Bond numbers $B_{d}=0$ and $B_{d}=1$. For the viscous drop, increasing the Bond number reduces the critical $\mathrm{Ca}$, while the inviscid drop shows exactly the opposite behaviour. Again, the explanation lies in the different behaviour of the viscous and pressure forces in the respective cases. While this phenomenon is significant for the large contact angle $\theta_{A}=90^{\circ}$, it is much more dramatic for a small contact angle such as $\theta_{A}=20^{\circ}$. In the asymptotic limits, the behaviour of the inviscid and viscous droplets diverges completely. For arbitrary contact angle, as the Bond number approaches infinity, the critical $\mathrm{Ca}$ for an inviscid droplet is infinity, while for a viscous droplet it is zero. For arbitrary Bond number, in the limit of small contact angles, the critical $\mathrm{Ca}$ for inviscid droplets approaches infinity, while that for viscous droplets approaches zero.

We end this section by presenting empirical equations which correlate the data for the critical $\mathrm{Ca}$ at non-zero Bond number. For the inviscid droplets, the critical $\mathrm{Ca}$ is correlated using equation (25), and coefficients for the data in figure $11(a)$ at $B_{d}=1$ yield

$$
B_{d}=1, \quad \lambda=0: \quad b_{0}=3.336, \quad b_{1}=-0.985, \quad b_{2}=0.173
$$

with a maximum relative error of $3 \%$. 

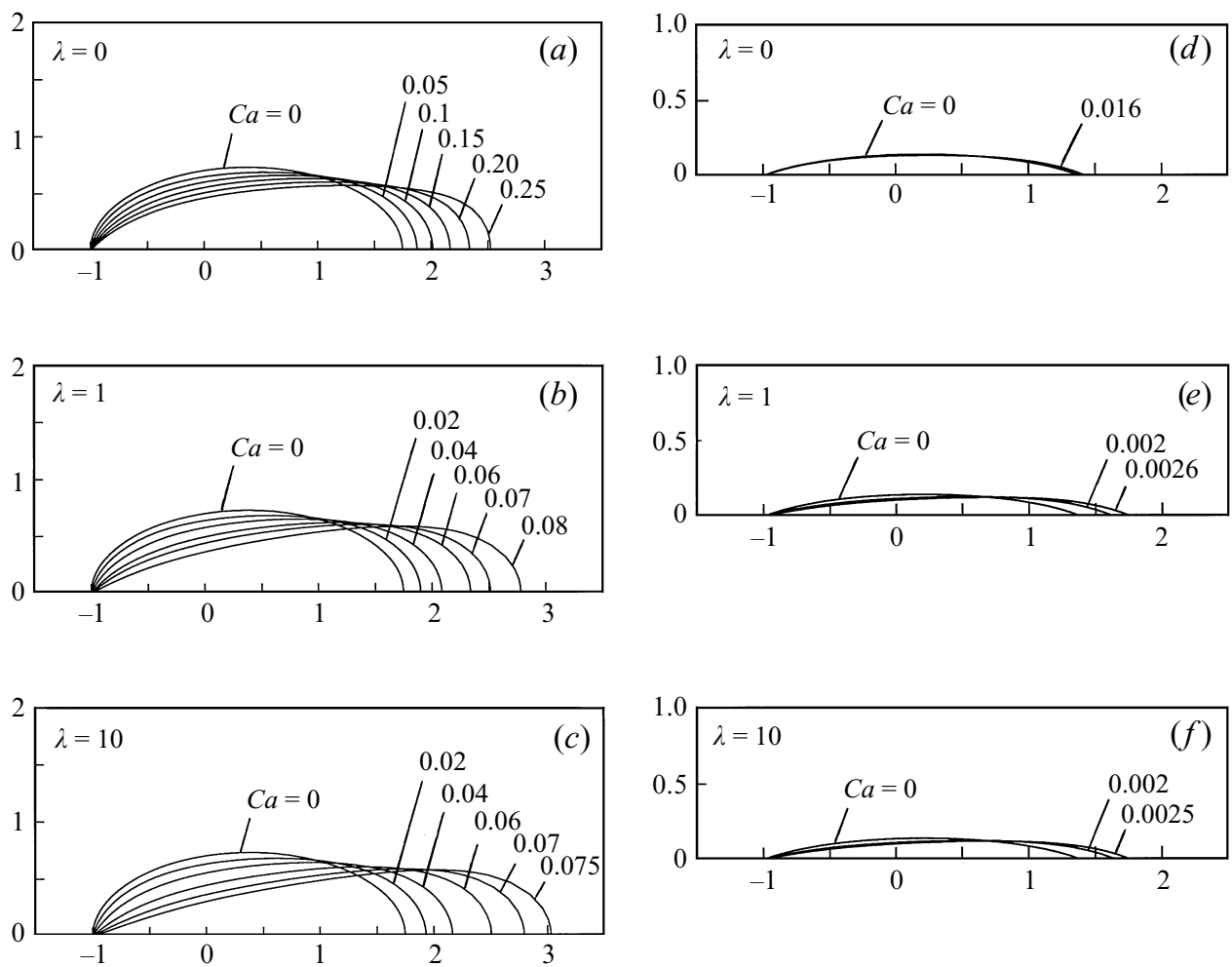

FIGURE 10. Equilibrium profiles for droplets in shear flows with Bond number $B_{d}=1$ for contact angles $\theta_{A}=90^{\circ}(a-c)$ and $\theta_{A}=20^{\circ}(d-f)$.

The critical capillary number for the viscous droplets is correlated by an equation of the form

$$
C a=\left(\frac{2}{27 \pi}\right)^{1 / 2} \frac{\left(\cos \theta_{R}-\cos \theta_{A}\right) \theta_{A}^{1 / 2}}{b_{0}+b_{1} \theta_{A}+b_{2}\left(\Delta \theta / \theta_{A}\right)} .
$$

The coefficient $b_{0}$ is a function of the Bond number only and is determined as an implicit function by the asymptotic results (A 16), (A 17). The coefficients $b_{1}$ and $b_{2}$ are determined by least-squares fits to the data. The results are:

$$
\left.\begin{array}{rrrrr}
B_{d}=1, & \lambda=1: & b_{0}=1.170, & b_{1}=0.457, & b_{2}=0.332, \\
B_{d}=1, & \lambda=10: & b_{0}=1.170, & b_{1}=0.676, & b_{2}=0.256, \\
B_{d}=5, & \lambda=1: & b_{0}=1.824, & b_{1}=0.401, & b_{2}=0.556 .
\end{array}\right\}
$$

The maximum relative error with this equation is $2 \%$ for $\lambda=1, B_{d}=1 ; 3 \%$ for $\lambda=10, B_{d}=1$ and $1.5 \%$ for $\lambda=1, B_{d}=5$.

\section{Conclusions}

In this paper we have conducted a comprehensive study of the drop displacement problem for two-dimensional droplets in viscous shear flows. We have considered a wide selection of parameters to investigate the physical behaviour of different droplets and to test the limits of theoretical predictions. This study complements the analytical theory of Dussan (1987) for displacement of droplets in low-Reynolds-number flows 

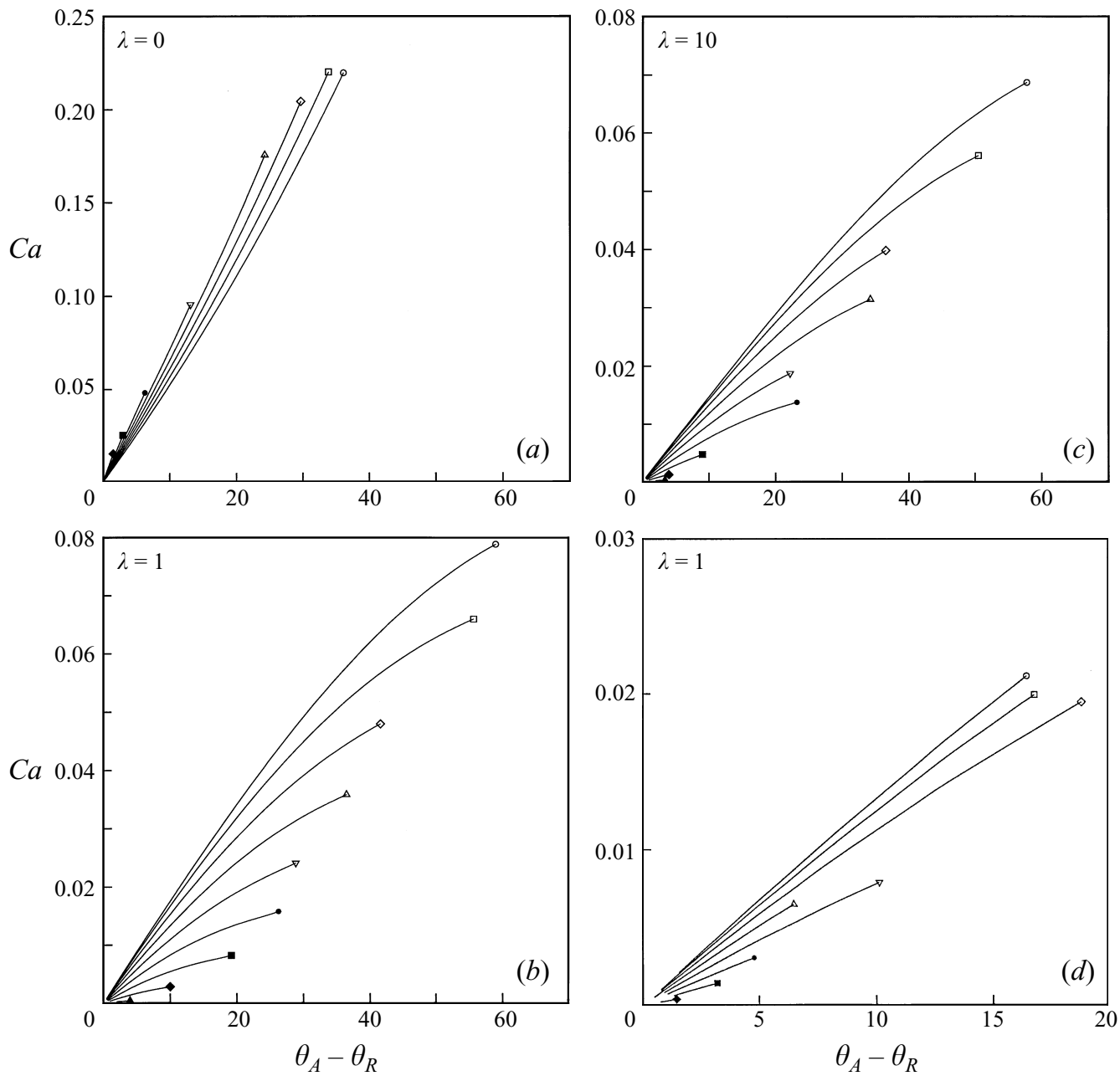

FIGURE 11. Critical $C a$ for droplets with gravity effects: $(a-c) B_{d}=1,(d) B_{d}=5$. Contact angles $\theta_{A}: \boldsymbol{\Delta}, 10^{\circ} ; \diamond, 20^{\circ} ; \mathbf{\square}, 30^{\circ} ; \bullet, 40^{\circ} ; \nabla, 50^{\circ} ; \triangle, 60^{\circ} ; \diamond, 70^{\circ} ; \square, 80^{\circ} ; \circ 90^{\circ}$

and provides a counterpoint to the numerical study of Feng \& Basaran which focused on the high Re inertial regime. A number of important conclusions have been reached.

(i) Asymptotic results based on lubrication theory should give correct qualitative predictions for the behaviour of viscous drops, however the range of validity is quite small and the quantitative predictions show significant errors. The critical capillary number shows significant departure from the linear dependence on hysteresis $\theta_{A}-\theta_{R}$ predicted by asymptotic theories.

(ii) Viscosity ratio plays an important role for viscous droplets, showing a significant effect at high contact angles. This sensitivity to viscosity ratio is not predicted by lubrication theory.

(iii) Inviscid droplets show dramatic and often contradictory behaviour compared to viscous droplets. These effects are most severe at high Bond number and small contact angles. Gas bubbles and liquid droplets may be expected to show dramatically different behaviour owing to these differences. 

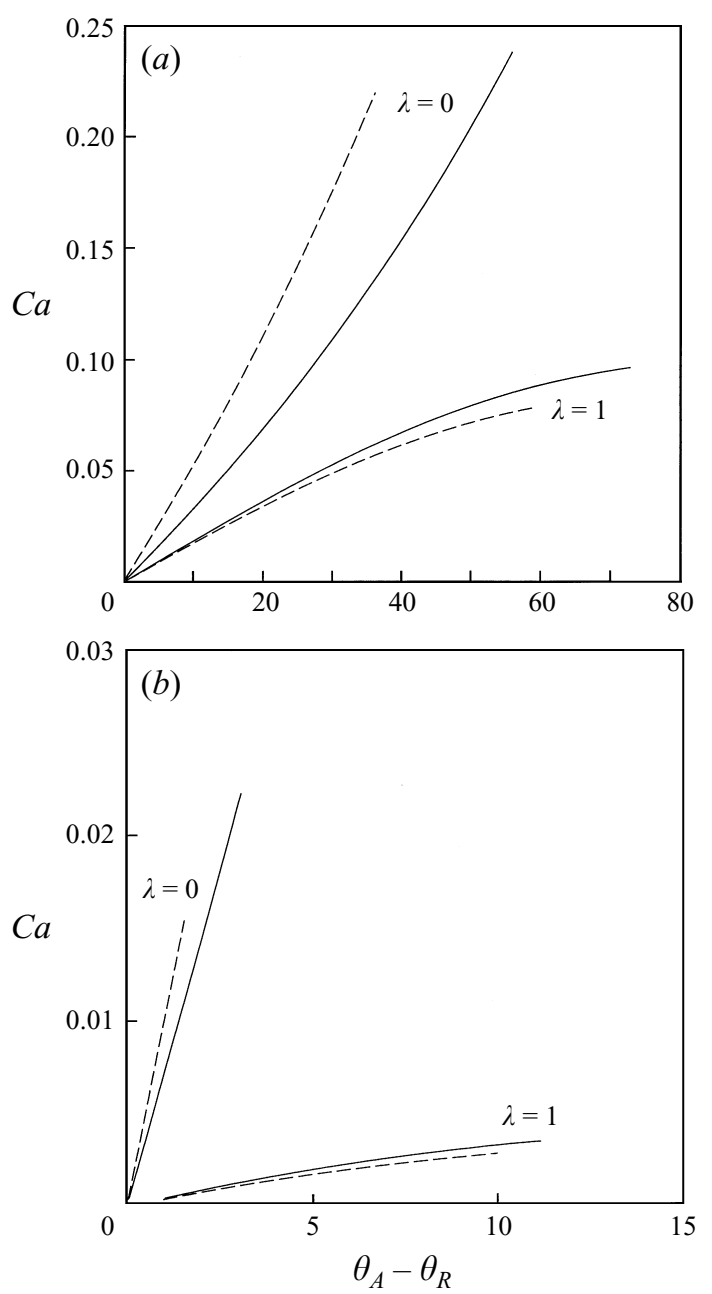

Figure 12. Critical $C a$ for droplets with different viscosity ratio and Bond numbers. $(a) \theta_{A}=90^{\circ}$, (b) $\theta_{A}=20^{\circ}$. -,$B_{d}=0 ;----, B_{d}=1$.

(iv) Gravitational effects may play an important role in the drop displacement process. The spreading effect associated with high Bond numbers reduces the ability of viscous drops to withstand fluid motion, but dramatically increases the resistance of inviscid droplets.

We close by noting that these results are based on fundamental physical principles associated with the scaling of viscous, pressure and surface forces. As a result, we anticipate that the conclusions will remain valid in the analogous problem for the displacement of three-dimensional droplets in viscous shear flow. The Newton iteration procedure introduced in this paper proved to be a robust and efficient numerical technique. In combination with the three-dimensional spectral element algorithm of Muldowney \& Higdon (1995), we believe it will prove well suited to equilibrium problems of this type.

This work was supported by the National Science Foundation. Computations were performed on IBM RS6000 workstations furnished with support from the IBM SUR program at the University of Illinois. 


\section{Appendix}

For droplets with small contact angles, $\theta_{R}, \theta_{A} \ll 1$, lubrication theory may be employed to reach a simple analytical result for the yield condition for drop displacement. This procedure is well known, and we cite directly those results which are required for the simple two-dimensional flows considered here. Dussan (1987) and Johnson (1981) include formal expositions for the associated three-dimensional and axisymmetric problems.

Let the droplet occupy the region $(-\ell, \ell)$ on the $x$-axis as described above, and let the drop surface be specified as $h(x)$. Let the velocity inside the droplet have components $(u, v)$, then the $x$-component of velocity is given by

$$
u=\frac{3 G}{4 \lambda h}\left(y^{2}-2 h y\right)+\frac{G}{\lambda} y .
$$

To leading order, the shear stress on the drop surface is simply $\tau_{\infty}=\mu G$. The given velocity satisfies this condition as well as the no-slip condition on the wall. The pressure gradient inside is determined by the condition of zero volume flow for a stationary droplet. Based on (A 1), the shear stress on the solid wall is $\tau=-\frac{1}{2} \tau_{\infty}$.

A momentum balance over the volume of the drop (including the interface) yields for the $x$-component

$$
2 \ell\left(\frac{3}{2} \tau_{\infty}\right)+\gamma \cos \theta_{A}-\gamma \cos \theta_{R}=0 .
$$

Note that the normal stress is of lower order and does not contribute to the leading order momentum balance in the $x$-direction. The momentum balance shows the importance of contact angle hysteresis in the drop displacement problem. If $\theta_{R}=\theta_{A}$, there is no net interfacial force to resist the fluid drag force. Given the $x$-momentum balance, the yield condition for the shear stress required to displace the droplet may be expressed in dimensionless form via the capillary number

$$
C a=\frac{1}{3}\left(\cos \theta_{R}-\cos \theta_{A}\right) \frac{a}{\ell}
$$

where we recall that $a$ is the equivalent radius based on the drop volume. To leading order for $\Delta \theta \ll \theta_{A}$, the effective radius is $a / \ell=(2 \theta / 3 \pi)^{1 / 2}$. Expanding the cos terms for small $\theta$, (A 3 ) becomes

$$
C a=\left(\frac{2}{27 \pi}\right)^{1 / 2} \theta_{A}^{3 / 2} \Delta \theta
$$

where $\Delta \theta=\theta_{A}-\theta_{R}$.

This result (A 4) may be compared with the theory of Dussan (1987) which predicts $\mathrm{Ca} \sim \theta_{A}^{4 / 3} \Delta \theta$ for three-dimensional drops.

It is interesting to note that the yield condition (A 4) is independent of the viscosity of the droplet to leading order. This result is valid for all viscosities except in the limit as $\lambda \rightarrow 0$. To illustrate the breakdown of the theory for small $\lambda$, we note that lubrication theory assumes a leading-order outer flow $u^{\infty}=G y$. Evaluating the velocity at the upper surface of the droplet, we find

$$
(u)_{y=h}=\frac{1}{4} \frac{G h}{\lambda} \sim G \ell \frac{\theta}{\lambda} .
$$

Comparing this to a characteristic velocity $G \ell$ in the outer flow, we require that $u \rightarrow 0$ as $\theta \rightarrow 0$ to assure that $u$ agrees with $u^{\infty}$. This condition is satisfied for all fixed $\lambda \neq 0$. On the other hand, consider some $\theta \ll 1$ for which $\theta \sim \lambda$. In this 
case, the velocity does not approach zero as $\theta \rightarrow 0$, and the outer flow does not reduce to a simple shear flow. The explicit form of the velocity $u_{0}(x)$ evaluated at $\theta \rightarrow 0$ can only be determined by solving the exterior boundary value problem with a boundary condition $u \sim \tau$ at the surface of the droplet. The proportionality coefficient is determined by the interior flow. In the absence of the detailed solution, the most we can say is that $u$ scales as $G \ell$ based on dimensional analysis. The velocity profile is now of the form

$$
u=\frac{u_{0}}{h^{2}}\left(3 y^{2}-2 h y\right)
$$

and the shear stresses on the droplet surface and on the wall are

$$
\tau_{h}=\frac{4 \lambda \mu u_{0}}{h} \quad \text { and } \quad \tau_{w}=\frac{-2 \lambda \mu u_{0}}{h} .
$$

With these results and the scaling $u_{0} \sim G \ell$, the total viscous force on the drop scales as

$$
\ell\left(\frac{\lambda \mu G \ell}{h}\right) \sim \ell\left(\frac{\lambda \mu G}{\theta}\right) \sim \ell\left(\frac{\lambda}{\theta} \tau_{\infty}\right) .
$$

Substituting this term for the viscous force in the momentum balance (A 2) yields

$$
C a \sim \frac{1}{\lambda} \theta_{A}^{5 / 2} \Delta \theta
$$

which implies that the critical $C a$ will increase rapidly as $\lambda$ decreases for drops whose viscosity is much smaller than the contact angle.

Proceeding a bit further, we consider an inviscid droplet with $\lambda \equiv 0$. For this case, the shear stress is exactly zero, and the only hydrodynamic force is the normal stress. The pressure inside the droplet is a constant, while the normal stress on the outer surface is dictated by the surface tension and the curvature. Owing to this fact, the momentum balance in the $x$-direction reduces to a simple identity. The prediction of the critical $\mathrm{Ca}$ would require the full solution for the outer pressure field. While we cannot achieve this goal analytically, a simple scaling analysis provides useful information. For small contact angles $\theta_{A}$ and $\Delta \theta / \theta_{A} \ll 1$, normal stresses responsible for a net force in the $x$-direction scale as $\left(\theta \tau_{\infty}\right)$ and the total force scales as

$$
F_{x}=\int_{-\ell}^{\ell} p n_{x} \mathrm{~d} x \sim\left(\theta \tau_{\infty}\right) \theta \ell \sim \ell \theta^{2} \tau_{\infty} .
$$

With this estimate for the normal stress, the critical capillary number scales as

$$
C a \sim \theta_{A}^{-1 / 2} \Delta \theta .
$$

The point of transition between the predictions (A 9) and (A 11) may be determined by comparing the relative magnitude of the shear stress estimate (A 8) with the normal stress (A 10). In summary, we have three different scalings for the critical capillary number, based on the value of the viscosity ratio $\lambda$ :

$$
\left.\begin{array}{rlrl}
\theta \ll \lambda: & & C a \sim \theta_{A}^{3 / 2} \Delta \theta, \\
\theta^{3} \leqslant \lambda \leqslant \theta: & & C a \sim \frac{1}{\lambda} \theta_{A}^{5 / 2} \Delta \theta, \\
\lambda<\theta^{3}: & & C a \sim \theta_{A}^{-1 / 2} \Delta \theta .
\end{array}\right\}
$$

The small-angle lubrication theory for viscous fluids is easily extended to include gravitational effects. The $x$-momentum balance (A 3) carries forward as before. To 
relate the effective radius $a$ to $\ell$, we need to solve for the drop profile. Employing the interfacial stress boundary condition with curvature $h^{\prime \prime}$ yields

$$
-\gamma h^{\prime \prime}=p_{0}+\left(\rho_{2}-\rho_{1}\right) g h .
$$

Integrating twice and employing the conditions $h( \pm \ell)=0, h^{\prime}(\ell) \approx-\theta_{A}$ we eliminate $p_{0}$ and find the drop profile

$$
h=\frac{\theta_{A}}{\beta \tanh \beta \ell}\left[1-\frac{\cosh \beta x}{\cosh \beta \ell}\right] .
$$

where $\beta^{2}=\left(\rho_{1}-\rho_{2}\right) g / \gamma$.

Integrating to find the volume of the drop yields the desired expression for $a / \ell$

$$
\frac{a}{\ell}=\left[\frac{2 \theta_{A}}{\pi} \frac{\beta \ell-\tanh \beta \ell}{(\beta \ell)^{2} \tanh \beta \ell}\right]^{1 / 2} .
$$

Combining this result with the momentum balance yields the critical $\mathrm{Ca}$ for drop displacement

$$
C a=\left(\frac{2}{9 \pi}\right)^{1 / 2} \theta_{A}^{3 / 2} \Delta \theta\left[\frac{\beta \ell-\tanh \beta \ell}{(\beta \ell)^{2} \tanh \beta \ell}\right]^{1 / 2} .
$$

The parameter $\beta \ell$ is related to the Bond number through $a / \ell$, hence

$$
B_{d}=\frac{2}{\pi} \frac{\beta \ell-\tanh \beta \ell}{\tanh \beta \ell} .
$$

Equations (A 16) and (A 17) give the critical capillary number as an implicit function of $B_{d}$. The functional relationship may be made clearer by considering expansions at the asymptotic limits. For $B_{d} \ll 1$, we have

$$
C a=\left(\frac{2}{27 \pi}\right)^{1 / 2} \theta_{A}^{3 / 2} \Delta \theta\left[1-\frac{\pi}{20} B_{d}+\frac{\pi^{2}}{1120} B_{d}^{2}\right],
$$

and for $B_{d} \gg 1$

$$
C a=\left(\frac{2}{3 \pi}\right) \theta_{A}^{3 / 2} \Delta \theta B_{d}^{-1 / 2} .
$$

If (A 18) is employed for $B_{d} \leqslant 3$ and (A 19) for higher values the predicted $C a$ is within $10 \%$ of the value given by (A 16), (A 17).

\section{REFERENCES}

Canuto, C., Hussaini, M. Y., Quarteroni, A. \& Zang, T. A. 1988 Spectral Methods in Fluid Dynamics. Springer.

Chen, Q., Rame, E. \& Garoff, S. 1995 The breakdown of asymptotic hydrodynamic models of liquid spreading at increasing capillary number. Phys. Fluids 7, 2631-2639.

Christodoulou, K. N. \& Scriven, L. E. 1992 Discretization of free surface flows and other moving boundary problems. J. Comput. Phys. 99, 39-55.

Dimitrakopoulos, P. 1996 Computational studies of droplet displacement in Stokes flow. MS thesis, University of Illinois.

Durbin, P. A. 1988 On the wind force needed to dislodge a drop adhered to a surface. J. Fluid Mech. 196, 205-222.

Dussan V., E. B. 1979 On the spreading of liquids on solid surfaces: static and dynamic contact lines. Ann. Rev. Fluid Mech. 11, 371-400. 
Dussan V., E. B. 1985 On the ability of drops or bubbles to stick to non-horizontal surfaces of solids. Part 2. Small drops or bubbles having contact angles of arbitrary size. J. Fluid Mech. 151, 1-20.

Dussan V., E. B. 1987 On the ability of drops to stick to surfaces of solids. Part 3. The influences of the motion of the surrounding fluid on dislodging drops. J. Fluid Mech. 174, 381-397.

Dussan V., E. B. \& Chow, R. T.-P. 1983 On the ability of drops or bubbles to stick to non-horizontal surfaces of solids. J. Fluid Mech. 137, 1-29.

FenG, J. Q. \& BASARAN, O. A. 1994 Shear flow over a translationally symmetric cylindrical bubble pinned on a slot in a plane wall. J. Fluid Mech. 275, 351-378.

Furmidge, C. G. L. 1962 Studies at phase interfaces: The sliding of liquid drops on solid surfaces and a theory for spray retention. J. Colloid Sci. 17, 309-324.

Gaydos, J. \& Neumann, A. W. 1987 The dependence of contact angles on drop size and line tension. J. Colloid Interface Sci. 120, 76-86.

GooD, R. J. 1979 Contact angles and the surface free energy of solids. In Surface and Colloid Science (ed. R. J. Good \& R. R. Stromberg). Plenum.

Good, R. J. \& Koo, M. N. 1979 The effect of drop size on contact angle. J. Colloid Interface Sci. 71, 283-292.

Higdon, J. J. L. 1985 Stokes flow in arbitrary two-dimensional domains: shear flow over ridges and cavities. J. Fluid Mech. 159, 195-226.

Higdon, J. J. L. \& Schnepper, C. A. 1994 High order boundary integral methods for viscous free surface flows. In Free Boundaries in Viscous Flows (ed. R. A. Brown \& S. H. Davis). Springer.

Johnson, R. E. 1981 Stokes flow past a sphere coated with a thin fluid film. J. Fluid Mech. 110, $217-238$

JoHnson, R. E. \& SADHAL, S. S. 1985 Fluid mechanics of compound multiphase drops and bubbles. Ann. Rev. Fluid Mech. 17, 289-320.

KING, A. C. \& TucK, E. O. 1993 Thin liquid layers supported by steady air-flow surface traction. J. Fluid Mech. 251, 709-718.

Kistler, S. F. 1993 Hydrodynamic wetting. In Wettability (ed. J. C. Berg). Dekker.

KistLeR, S. F. \& SCRIVEN, L. E. 1984 a Coating flow theory by finite element and asymptotic analysis of the Navier-Stokes system. Intl J. Numer. Meth. Fluids 4, 207-229.

Kistler, S. F. \& Scriven, L. E. $1984 b$ Coating flows. In Computational Analysis of Polymer Processing (ed. J. R. A. Pearson \& S. M. Richardson). Applied Science Publishers.

Li, X. \& Pozrikidis, C. 1996 Shear flow over a liquid drop adhering to a solid surface. J. Fluid Mech. 307, 167-190.

Marmur, A. 1994 Contact angle hysteresis on heterogeneous smooth surfaces. J. Colloid Interface Sci. 168, 40-46.

Muldowney, G. P. \& Higdon, J. J. L. 1995 A spectral boundary element approach to threedimensional Stokes flow. J. Fluid Mech. 298, 167-192.

Occhialini, J. M., Muldowney, G. P. \& Higdon, J. J. L. 1992 Boundary integral/spectral element approaches to the Navier-Stokes equations. Intl J. Numer. Meth. Fluids 15, 1361-1381.

Oliver, J. F., Huh, C. \& Mason, S. G. 1977 Resistance to spreading of liquids by sharp edges. J. Colloid Interface Sci. 59, 568-581.

PozRIKIDIS, C. 1992 Boundary Integral and Singularity Methods for Linearized Viscous Flow. Cambridge University Press.

Richardson, S. 1968 Two-dimensional bubbles in slow viscous flows. J. Fluid Mech. 33, 475-493.

Richardson, S. 1973 Two-dimensional bubbles in slow viscous flows. Part 2. J. Fluid Mech. 58, $115-127$.

Saito, H. \& Scriven, L. E. 1981 Study of coating flow by the finite element method. J. Comput. Phys. 42, 53-76.

Tuck, E. O. \& Schwartz, L. W. 1991 Thin static drops with a free attachment boundary. J. Fluid Mech. 223, 313-324.

Yekta-Fard, M. \& Ponter, A. B. 1988 The influences of vapor environment and temperature on the contact angle-drop size relationship. J. Colloid Interface Sci. 126, 134-140. 